\title{
Interaction of plane gravitational waves with a Fabry-Perot cavity in the local Lorentz frame
}

\author{
Sergey P. Tarabrin \\ Faculty of Physics, Moscow State University, Moscow, 119992, Russid
}

(Dated: October 13, 2018)

\begin{abstract}
We analyze the interaction of plane '+'-polarized gravitational waves with a Fabry-Perot cavity in the local Lorentz frame of the cavity input mirror outside of the range of long-wave approximation with the force of radiation pressure taken into account. The obtained detector response signal is represented as a sum of two parts: (i) the phase shift due to displacement of the movable mirror under the influence of gravitational wave and the force of light pressure, and (ii) the phase shift due to direct interaction of gravitational wave with light wave inside the cavity. We obtain formula for the movable mirror law of motion paying close attention to the phenomena of optical rigidity, radiative friction and direct coupling of gravitational wave to light wave. Some issues concerning the detection of high-frequency gravitational waves and the role of optical rigidity in it are discussed. We also examine in detail special cases of optical resonance and small detuning from it and compare our results with the known ones.
\end{abstract}

PACS numbers: 04.30.Nk, 04.80.Nn, 07.60.Ly, 95.55.Ym

\section{INTRODUCTION}

The search for gravitational waves (GWs) is now conducted with large Earth-based Michelson-type laser interferometers where test masses are separated by a distance of several kilometers [1, 2, 3]. Variations in the proper distances between these test masses are measured with the light wave. Traditionally interferometric GW detectors are analyzed in the transverse-traceless (TT) gauge, where coordinate lines are chosen to coincide with the geodesics of the freely falling test masses (if initial velocities of test masses are equal to zero) [4, [5]. This means that the coordinate frame is rigidly bounded with the $i n$ ertial test masses. For a '+'-polarized plane gravitational wave metric in the TT gauge has the usual linearized form [6]:

$$
\begin{aligned}
d s^{2} & =g_{\alpha \beta}(x) d x^{\alpha} d x^{\beta}=\left[\eta_{\alpha \beta}+h_{\alpha \beta}(x)\right] d x^{\alpha} d x^{\beta} \\
& =-c^{2} d t^{2}+(1+h) d x^{2}+(1-h) d y^{2}+d z^{2} .
\end{aligned}
$$

Here $h=h(t-z / c),|h| \ll 1$ is the GW wavefunction, $\eta_{\alpha \beta}=\operatorname{diag}(-1,1,1,1)$ is the metric tensor of flat spacetime, and $h_{\alpha \beta}$ are small deviations from it. Greek indices run over $0,1,2,3$ or $c t, x, y, z$; Latin indices run over $1,2,3$ or $x, y, z$.

Geodesic equation in metric (1) dictates that the test mass coordinates are not changed by gravitational wave [5], i.e. GW does not interact with the test masses in the TT gauge. Nevertheless, it noticeably changes the phase of electromagnetic wave (EMW for short) which propagates in the region of space-time where gravitational wave is present. The elementary solution of eikonal equation for a single arm of laser interferometer [5] states that the phase shift is proportional to GW

*Electronic address: tarabrin@phys.msu.ru signal: $\delta \Psi(t)=k_{0} L h(t)$, where $k_{0}$ is the wavenumber of electromagnetic wave and $L$ is the interferometer arm length. In other words one can reduce the analysis of a '+'-polarized GW interaction with detector to the problem of EMW propagation in the medium with effective refraction index $n^{2}(t)=1+h(t)$. This result is only valid in long-wave approximation $L \ll \lambda_{\mathrm{gw}}$, where $\lambda_{\mathrm{gw}}$ is the length of gravitational wave. In general case the formula for phase shift is more complex but it is still the solution of the eikonal equation. The significant advantage of the TT gauge is the ease of going from long-wave approximation to the general case [5].

However, the crucial requirement of the test masses inertiality cannot be met in the real Earth-based experiments since there exists a great amount of nongravitational forces acting on the test masses, such as seismic and thermal fluctuating forces, light pressure resulting in ponderomotive rigidity $7,68,9,10,11,12,13$, 14, 15, 16, 17] and radiative friction [18]. The latter two phenomena appear if Fabry-Perot (FP) cavities are utilized in the interferometer as in LIGO and VIRGO detectors [3]. Therefore, the reference frame of the TT gauge can hardly be constructed for the Earth-based detectors. Correspondingly, the results obtained within the bounds of the TT gauge are only applicable when condition of the test masses inertiality is met (the case of space GW antenna LISA [19, 20] in which drag-free technique will be used). Otherwise, additional analysis should be performed in order to validate the TT-based consideration of non-geodesic motion. Clearly, this will significantly complicate the whole problem.

In order to get rid of inertiality requirement when analyzing the interaction of GW with a Fabry-Perot cavity and take non-gravitational forces into account we should work in the local Lorentz frame (LL gauge) of the cavity input mirror. Coordinate transformation from the TT gauge to the LL gauge can be found in Ref. [5]:

$$
d s^{2}=g_{\alpha \beta}(x) d x^{\alpha} d x^{\beta}=-c^{2} d t^{2}+d x^{2}+d y^{2}+d z^{2}
$$




$$
+\frac{1}{2 c^{2}}\left(x^{2}-y^{2}\right) \ddot{h}(c d t-d z)^{2},
$$

where old notations for the coordinates are used since we are not interested in the coordinates of the TT gauge anymore.

A simple analysis performed in Ref. [5] shows that in the LL gauge gravitational wave mainly interacts with the test masses, resulting in the phase shift $\delta \Psi(t)=$ $2 k_{0} \delta X(t)=k_{0} L h(t)$, while direct interaction with the light wave leads only to a small correction in long-wave approximation. Here $\delta X(t)=(1 / 2) L h(t)$ is the displacement of the test mass due to its interaction with gravitational wave. In other words one can reduce the analysis of a '+'-polarized GW interaction with detector to the problem of EMW propagation in the volume without effective medium (in long-wave approximation only!) but with movable boundaries.

However, the LL gauge has one great disadvantage the complexity of going from long-wave approximation to the general case. In this paper we are mostly interested in the latter because still little is known about the particular features of non-inertial test masses motion under the influence of gravitational wave when condition $L \ll \lambda_{\mathrm{gw}}$ is violated. In this sense we are mostly interested in the phenomenon of optical rigidity (or optical spring) and its role in detection of high frequency GW signals. It is most likely that the optical strings will be implemented in the future generations of the Earth-based detectors (such as Advanced LIGO), allowing significant amplification of the response to GW signals. General formulas for optical rigidity, test masses motion and detector response signal outside of the range of long-wave approximation were obtained in Ref. [11]. Starting from the ideas of Ref. 21] we propose an independent derivation of general formulas and obtain a good agreement with results of Ref. [11] on the one hand, and of [12, 18] on the other. Our calculations allow us to take into account the relativistic correction to the force of radiation pressure, known as radiative friction [18], which was not obtained in Ref. [11]. Using modern notations we further analyze the GW- and radiation pressure-driven motion of test masses, paying close attention to general relativistic aspects of the problem. We also study in detail the differences between the general case and its long-wave approximation. It should be noted that we deal only with the optimal GW polarization and detector orientation ('+'-polarized GW with one of its principal axes parallel to the axis of FP cavity) since it is enough for consideration of the radiation pressure-induced dynamics.

Motivation for our work is the following. Modern GW detectors, utilizing Fabry-Perot cavities, with arm lengths of several kilometers ( $4 \mathrm{~km}$ for LIGO and $3 \mathrm{~km}$ for VIRGO) are best suited for detection of GW signals with frequencies that lie in the region of $10^{2}$ to $10^{3} \mathrm{~Hz}$. For them $L / \lambda_{\mathrm{gw}} \sim 10^{-3} \div 10^{-2} \ll 1$ and long-wave approximation holds with high accuracy. Today the most efforts are made to detect these low-frequency signals which are emitted, for example, by binary pulsars, rotating neutron stars, supernova explosions etc. However, modern astrophysical and cosmological theories predict the existence of sources of high-frequency $\left(f \geq 10^{4} \mathrm{~Hz}\right) \mathrm{GW}$ signals, such as relic gravitational wave background [22, 23, 24]. Some models, such as inflation, predict the GW spectrum of the background with typical values of amplitude which are far beyond the sensitivity of even advanced interferometers. However, the spectrum of gravitational waves predicted by string cosmology [25, 26, 27] has rather big typical GW amplitude which rises with increasing frequency, thus allowing the potential possibility of detection of GWs with certain frequencies above $10^{3} \mathrm{~Hz}$ on the Earth-based detectors. There is a strong evidence that modern and future detectors are (or will be) capable of detecting signals at frequencies about $10^{4} \mathrm{~Hz}$. For example, in Refs. [28, 29] (see also Refs. [30, 31]) it was proposed that $4 \mathrm{~km}$ LIGO detectors could detect 37.5 $\mathrm{kHz}$ signals, corresponding to cavity free spectral range (FSR), for which $L / \lambda_{\mathrm{gw}}=1 / 2$, thus operating outside of long-wave regime. And though in this case the response signal vanishes for optimal polarization of gravitational wave 32], GW signals can still be detected with enhanced sensitivity at multiples of the cavity FSR for other orientations. However, the role of radiation pressure effects in the detection of high-frequency GWs when condition $L / \lambda_{\mathrm{gw}} \ll 1$ is violated (especially in the vicinity of FSR and its multiples) has not been recognized up to date. We propose the study of these effects, since the searches for GW background are planned for the Advanced LIGO stage where optical springs will be utilized.

This paper is organized as follows. In Sec. II we write down the wave equation for electromagnetic wave propagating in the space-time of plane gravitational wave and obtain its solution for an arbitrary $h(t)$. In Sec. III we examine the special case of monochromatic $h(t)$. In Sec. IV] we apply these results to consideration of the boundary problem for EMW in a Fabry-Perot cavity with the movable mirror and obtain its response signal to GWs. By neglecting the resonant features of FP cavity in Sec. V we find excellent agreement between our LL-based calculations and TT-based calculations performed in Ref. [5] for the case of single reflection. In Sec. VI we write down full equation of motion for the mirror paying close attention to the phenomena of optical rigidity, radiative friction and GR corrections to the force of radiation pressure. In Sec. VII we find the mirror law of motion and substitute it into the response signal. The obtained formulas basically agree with results of Ref. [11]. We also discuss the possibility of parametric excitation of additional optical modes under the influence of GW signals with frequencies near cavity FSR and its multiples. In Sec. VIII we examine special cases of optical resonance and small detuning from it in general case and long-wave approximation. Excellent agreement with the results obtained in Refs. [12, 18] is found. In the end we estimate the deposit of optical rigidity into the response signal in the vicinity of FSR. 


\section{WAVE EQUATION AND ITS SOLUTION}

We start from the second pair of Maxwell's equations without sources in curved space-time [33]:

$$
\frac{1}{\sqrt{-g}} \frac{\partial}{\partial x^{\beta}}\left(\sqrt{-g} F^{\alpha \beta}\right)=0 .
$$

Here $F_{\mu \nu}=\partial_{\mu} A_{\nu}-\partial_{\nu} A_{\mu}, A^{\mu}=\left(A^{0}, A^{1}, A^{2}, A^{3}\right)$ is the 4-potential of electromagnetic field, and $g=\operatorname{det}\left(g_{\mu \nu}\right) \equiv$ -1 . Let us impose the Coulomb gauge $A^{0}=0, \partial_{1} A^{1}+$ $\partial_{2} A^{2}+\partial_{3} A^{3}=0$. Then

$$
g^{\alpha \beta} \partial_{\alpha} \partial_{\beta} A^{\mu}=0 .
$$

In general $A^{\mu}=A^{\mu}(x, y, z, t)$. Let us restrict ourselves to consideration of gravitational waves with frequencies $f_{\mathrm{gw}} \sim 10^{2} \div 10^{5} \mathrm{~Hz}$, corresponding to wavelengths $\lambda_{\text {gw }} \sim 10^{3} \div 10^{6} \mathrm{~m}$. This is far larger than the optical wavelength $\lambda_{0} \approx 1 \mathrm{mkm}$ and the radius of the laser beam $r \approx 10 \mathrm{~cm}$. For simplicity we assume that $A^{\mu}$ describes a linearly $z$-polarized plane light wave traveling along the $x$-axis. Therefore, under all listed conditions we have $A^{\mu}=(0,0,0, A)$ with $A=A(x, t)$ and the following scalar wave equation:

$$
\frac{\partial^{2} A}{\partial x^{2}}-\frac{1}{c^{2}} \frac{\partial^{2} A}{\partial t^{2}}=\frac{1}{2} \frac{x^{2}}{c^{2}} \ddot{h}(t) \frac{1}{c^{2}} \frac{\partial^{2} A}{\partial t^{2}} .
$$

The right side of this equation describes the direct interaction of gravitational wave with light wave [5]. In Ref. [21] this effect was called the distributed gravitational redshift.

It is convenient to solve Eq. (3) using the method of successive approximations since $h \ll 1$. We shall keep only the zeroth and the first order in $h$ terms: $A(x, t)=A^{(0)}(x, t)+A^{(1)}(x, t),\left|A^{(1)}\right| \sim\left|h A^{(0)}\right| \ll\left|A^{(0)}\right|$. The solution of the zeroth order is the sum of plane monochromatic waves traveling in positive and negative directions of the $x$-axis. We denote "positive" wave with index '+' and "negative" wave with index '-':

$$
\begin{aligned}
& A^{(0)}(x, t)=A_{+}^{(0)}(x, t)+A_{-}^{(0)}(x, t), \\
& A_{ \pm}^{(0)}(x, t)=A_{ \pm 0} e^{-i\left(\omega_{0} t \mp k_{0} x\right)}+\text { c.c. }
\end{aligned}
$$

where $k_{0}=\omega_{0} / c$. Amplitude and frequency are derived from some initial and boundary problems and we shall keep them undefined until Sec. IV] The first order equation is:

$$
\frac{\partial^{2} A^{(1)}}{\partial x^{2}}-\frac{1}{c^{2}} \frac{\partial^{2} A^{(1)}}{\partial t^{2}}=\frac{1}{2} \frac{x^{2}}{c^{2}} \ddot{h}(t) \frac{1}{c^{2}} \frac{\partial^{2} A^{(0)}}{\partial t^{2}} .
$$

The general solution of this equation can be represented as the sum of "positive" and "negative" waves:

$$
A^{(1)}(x, t)=A_{+}^{(1)}(x, t)+A_{-}^{(1)}(x, t) .
$$

Clearly, they can be treated independently. Remind, that $g_{00}(0, t)=-1$ and therefore, we must demand that

$$
A_{+}^{(1)}(0, t)=A_{-}^{(1)}(0, t)=0 .
$$

Physically these initial conditions mean that both the light waves $A_{ \pm}(x, t)$ experience no redshift at $x=0$, i.e. the solution of full Eq. (3) at $x=0$ is $A_{ \pm}(0, t)=$ $A_{ \pm}^{(0)}(0, t)=A_{ \pm 0} e^{-i \omega_{0} t}+A_{ \pm 0}^{*} e^{i \omega_{0} t}$. The solution of the Cauchy problem (5,7) is obtained in Appendix A

$$
\begin{aligned}
& A_{ \pm}^{(1)}(x, t)=A_{ \pm 0} g_{ \pm}(x, t) e^{-i\left(\omega_{0} t \mp k_{0} x\right)}+\text { c.c. } \\
& g_{ \pm}(x, t)=\int_{-\infty}^{+\infty} g_{ \pm}\left(x, \Omega+\omega_{0}\right) e^{-i \Omega t} \frac{d \Omega}{2 \pi}
\end{aligned}
$$

where

$$
\begin{aligned}
& g_{ \pm}(x, \Omega) \equiv \frac{1}{2} h\left(\Omega-\omega_{0}\right) \\
& \quad \times\left\{x^{2} \xi(\Omega) \mp i x \eta(\Omega)-\zeta(\Omega)\left[1-e^{\mp i\left(k_{0}-\frac{\Omega}{c}\right) x}\right]\right\} .
\end{aligned}
$$

and

$$
\begin{aligned}
& \xi(\Omega) \equiv \frac{\omega_{0}^{2}}{c^{2}} \frac{\Omega-\omega_{0}}{\Omega+\omega_{0}}, \\
& \eta(\Omega) \equiv 4 \frac{\omega_{0}}{c} \frac{\omega_{0}^{2}}{\left(\Omega+\omega_{0}\right)^{2}}, \\
& \zeta(\Omega) \equiv 2 \frac{\omega_{0}^{2}\left(\Omega^{2}+3 \omega_{0}^{2}\right)}{\left(\Omega-\omega_{0}\right)\left(\Omega+\omega_{0}\right)^{3}} .
\end{aligned}
$$

\section{CASE OF MONOCHROMATIC GRAVITATIONAL WAVE}

In order to make clear the physical meaning of the obtained result we shall examine the special case of monochromatic gravitational wave $h(\Omega)=2 \pi h_{0} \delta(\Omega-$ $\left.\omega_{\mathrm{gw}}\right)+2 \pi h_{0} \delta\left(\Omega+\omega_{\mathrm{gw}}\right)$, corresponding to $h(t)=$ $2 h_{0} \cos \omega_{\mathrm{gw}} t$ in time domain. We shall restrict ourselves to consideration of the light wave traveling in positive direction of the $x$-axis and use natural requirement $\omega_{\text {gw }} / 2 \pi \sim 10^{2} \div 10^{5} \mathrm{~Hz} \ll \omega_{0} / 2 \pi \sim 10^{14} \mathrm{~Hz}$. Under these conditions formulas (4) and (8) lead to:

$$
\begin{aligned}
& A_{+}(x, t)=\mathcal{A}_{+}(x, t) e^{i\left(k_{0} x-\omega_{0} t\right)} e^{i \Psi_{+}(x, t)} \\
& =A_{+0}\left\{1-i h_{0}\left[a(x) \sin \omega_{\mathrm{gw}} t+b(x) \cos \omega_{\mathrm{gw}} t\right.\right. \\
& \left.\left.+\frac{\omega_{0}}{\omega_{\mathrm{gw}}} \sin \omega_{\mathrm{gw}}\left(t-\frac{x}{c}\right)\right]\right\} e^{i\left(k_{0} x-\omega_{0} t\right)}+\text { c.c. }
\end{aligned}
$$

where

$$
a(x) \equiv \frac{1}{2} \frac{x^{2}}{c^{2}} \omega_{0} \omega_{\mathrm{gw}}-\frac{\omega_{0}}{\omega_{\mathrm{gw}}}, \quad b(x) \equiv \frac{x}{c} \omega_{0} .
$$

We can now easily extract information about the amplitude and the phase of light wave. Up to the second order of $h$ amplitude remains unchanged $\mathcal{A}_{+}(x, t)=A_{+0}$ while phase $\Psi_{+}(x, t)$ is influenced by GW in the first order:

$$
\Psi_{+}(x, t)=-h_{0}\left[\left(\frac{x^{2}}{c^{2}} \omega_{0} \omega_{\mathrm{gw}}-\frac{\omega_{0}}{\omega_{\mathrm{gw}}}\right) \sin \omega_{\mathrm{gw}} t\right.
$$




$$
\left.+\frac{x}{c} \omega_{0} \cos \omega_{\mathrm{gw}} t+\frac{\omega_{0}}{\omega_{\mathrm{gw}}} \sin \omega_{\mathrm{gw}}\left(t-\frac{x}{c}\right)\right] .
$$

We must put $t=x / c$ if we are interested in the phase of light wave at point $x$. This is enough since taking into account the influence of gravitational wave on time will lead to the term of the second order in $h$.

$$
\begin{aligned}
\Psi_{+}(x, x / c)=-h_{0}\left[\left(\frac{x^{2}}{c^{2}} \omega_{0} \omega_{\mathrm{gw}}\right.\right. & \left.-\frac{\omega_{0}}{\omega_{\mathrm{gw}}}\right) \sin \omega_{\mathrm{gw}} \frac{x}{c} \\
& \left.+\frac{x}{c} \omega_{0} \cos \omega_{\mathrm{gw}} \frac{x}{c}\right] .
\end{aligned}
$$

We plotted the graph of this function in Fig. (1).

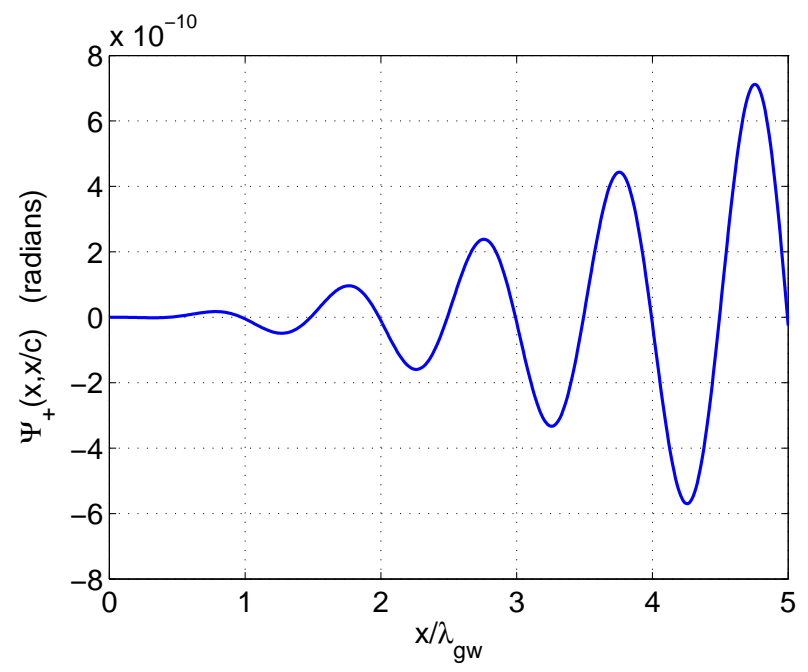

FIG. 1: Phase shift $\Psi_{+}(x, x / c)$ acquired by "positive" wave from its direct interaction with monochromatic gravitational wave. For visualization we assumed $\omega_{0} / 2 \pi=3 \times 10^{14} \mathrm{~Hz}$, $\omega_{\mathrm{gw}} / 2 \pi=37.5 \mathrm{kHz}, h_{0}=10^{-22}$.

The long-wave approximation $x / \lambda_{\text {gw }} \ll 1$ of this formula is

$$
\begin{aligned}
\Psi_{+}(x) & \approx-\frac{2(2 \pi)^{2}}{3} k_{0} x h_{0} \frac{x^{2}}{\lambda_{\mathrm{gw}}^{2}}+o\left(k_{0} x h_{0} \frac{x^{2}}{\lambda_{\mathrm{gw}}^{2}}\right) \\
& =O\left(k_{0} x h_{0} \frac{x^{2}}{\lambda_{\mathrm{gw}}^{2}}\right) .
\end{aligned}
$$

This result coincides with the one of Ref. [5]: the direct interaction of gravitational wave and light wave leads only to a small correction of the order of $\left(x / \lambda_{\mathrm{gw}}\right)^{2} h$ in the LL gauge. Note that formula (10) can be qualitatively interpreted as the propagation of light wave in effective medium with refraction index $n^{2}(x, t)=1+\delta n^{2}(x, t)$, where $\delta n(x, t) \propto(x / c)^{2} \ddot{h}(t), x \ll \lambda_{\mathrm{gw}}$.

\section{LIGHT WAVE IN A FABRY-PEROT CAVITY UNDER THE INFLUENCE OF PLANE GRAVITATIONAL WAVE}

Let us consider now the light wave traveling inside the Fabry-Perot cavity placed in the space-time of a plane '+'-polarized gravitational wave. The cavity is formed by two mirrors (see Fig. 2). The first one (left) is called the input mirror; it is immovable in its own reference frame (remind that we work in the local Lorentz frame of this mirror) and partially transmits optical radiation with amplitude coefficient $T \ll 1$. The second mirror the end-mirror - is movable and absolutely reflective. For simplicity we assume that both mirrors are ideal, meaning that they do not absorb or dissipate optical energy. We put distance between the mirrors in the absence of gravitational wave and optical radiation to be equal to $L$.

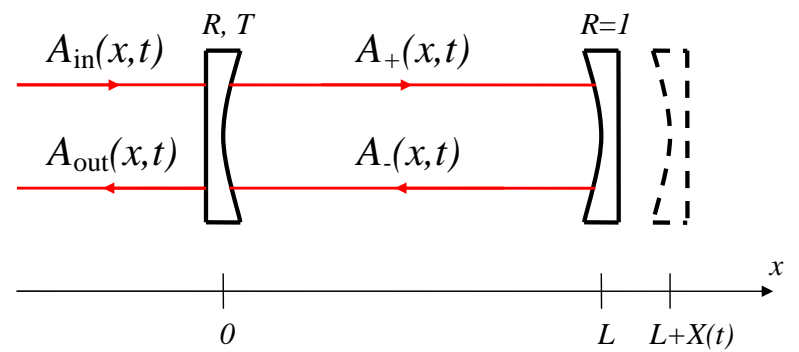

FIG. 2: Fabry-Perot cavity with the movable mirror. Input wave is denoted by index 'in', output wave is 'out'. Optical field inside the cavity is a sum of two waves running in two opposite directions of the $x$-axis: "positive" wave is denoted by index '+' and "negative" is '-'. Isolated cavity without any gravitational or optical radiation present has a fixed length $L$. Coordinate of the movable mirror is $L+X(t), X \ll L$.

Let the plane linearly polarized light wave $A_{\text {in }}(x, t)$ be incident on the input mirror. We shall write it down as a sum of "strong" wave with amplitude $A_{\text {ino }}$ and frequency $\omega_{0}$ and "weak" addition $a_{\text {in }}(x, t)$ which corresponds to the noises of light field:

$$
\begin{aligned}
& A_{\text {in }}(x, t)=A_{\text {in } 0} e^{-i\left(\omega_{0} t-k_{0} x\right)}+a_{\text {in }}(x, t) e^{-i\left(\omega_{0} t-k_{0} x\right)}+\text { c.c. }, \\
& a_{\text {in }}(x, t)=\int_{-\infty}^{+\infty} a_{\text {in }}\left(\Omega+\omega_{0}\right) e^{-i \Omega\left(t-\frac{x}{c}\right)} \frac{d \Omega}{2 \pi} .
\end{aligned}
$$

For simplicity in this paper we consider classical EM fields (and corresponding classical noises), since we are focused on dynamics only. The generalization for quantized EM fields is straightforward: classical field amplitudes should be replaced with corresponding annihilation operators and complex conjugates - with Hermitian conjugates.

We represent the optical field inside the cavity as a sum of two light waves: the one $A_{+}(x, t)$ running in the positive direction of the $x$-axis and another one $A_{-}(x, t)$ 
running in the opposite direction. We write them down as sums of three parts. The first part is the "strong" wave with amplitude $A_{ \pm 0}$. The second part is the explicit formula (8) for direct coupling 'GW+EMW'. The third part is the "weak" addition $a_{ \pm}(x, t)$ - the unknown function of its arguments representing both optical noises and phase shift acquired while circulating inside the cavity.

$$
\begin{aligned}
A_{ \pm}(x, t)= & A_{ \pm 0} e^{-i\left(\omega_{0} t \mp k_{0} x\right)}+A_{ \pm 0} g_{ \pm}(x, t) e^{-i\left(\omega_{0} t \mp k_{0} x\right)} \\
& +a_{ \pm}(x, t) e^{-i\left(\omega_{0} t \mp k_{0} x\right)}+\text { c.c. } \\
a_{ \pm}(x, t)= & \int_{-\infty}^{+\infty} a_{ \pm}\left(\Omega+\omega_{0}\right) e^{-i \Omega\left(t \mp \frac{x}{c}\right)} \frac{d \Omega}{2 \pi}
\end{aligned}
$$

We assume interaction between a gravitational wave $h$ and "weak" field $a_{ \pm}$to be a negligible effect since (i) it is most likely that coupling of GWs to optical noises does not contain any useful information and (ii) coupling of GWs to phase shift (variation of optical field) acquired due to GW itself leads to the negligible $h^{2}$ terms.

Output wave $A_{\text {out }}(x, t)$ includes the wave reflected from the input mirror and the wave flowing out of the cavity. The latter one carries information about gravitational wave (signal wave) along with optical noises. Again we represent the wave as a sum of "strong" and "weak" components:

$$
\begin{aligned}
A_{\text {out }}(x, t)= & A_{\text {out } 0} e^{-i\left(\omega_{0} t+k_{0} x\right)}+a_{\text {out }}(x, t) e^{-i\left(\omega_{0} t+k_{0} x\right)} \\
& + \text { c.c. }, \\
a_{\text {out }}(x, t) & =\int_{-\infty}^{+\infty} a_{\text {out }}\left(\Omega+\omega_{0}\right) e^{-i \Omega\left(t+\frac{x}{c}\right)} \frac{d \Omega}{2 \pi}
\end{aligned}
$$

Our problem is to find connection between $\quad A_{ \pm 0}, \quad A_{\text {out } 0}, \quad a_{ \pm}(x, t), \quad a_{\text {out }}(x, t) \quad$ and $A_{\text {in } 0}, a_{\text {in }}(x, t), \quad g_{ \pm}(x, t)$. This connection is derived from the boundary conditions. The first boundary condition is the requirement of vector potential continuity along the input mirror:

$$
\begin{aligned}
A_{\text {out }}(0, t) & =R A_{\text {in }}(0, t)+T A_{-}(0, t), \\
A_{+}(0, t) & =T A_{\text {in }}(0, t)-R A_{-}(0, t) .
\end{aligned}
$$

The second condition is the requirement of electric field to be equal to zero on the surface of end-mirror in its local reference frame 34]: $E_{z}^{\prime}(0, t-\tau)=0$ (we neglect $\dot{X}^{2} / c^{2}$ terms), where notation $\tau=L / c$ is introduced. Using well-known formulas for Lorentz transformations of electric and magnetic fields from movable frame of reference to the laboratory frame [33] we rewrite this condition in the following form:

$$
E_{z}(L+X, t-\tau)+(\dot{X} / c) H_{y}(L+X, t-\tau)=0,
$$

or, substituting here definitions $E_{z}=-(1 / c)(\partial A / \partial t)$ and $H_{y}=-\partial A / \partial x$, we come to the following equation:

$$
-\frac{1}{c}\left[\frac{\partial A(L+X, t-\tau)}{\partial t}+\dot{X} \frac{\partial A(L+X, t-\tau)}{\partial x}\right]=0
$$

which means that directional derivative of vectorpotential equals to zero along the mirror trajectory. Thus it is sufficient to demand $A(L+X, t-\tau)=0$ or

$$
A_{+}(L+X, t-\tau)+A_{-}(L+X, t-\tau)=0 .
$$

Here $X=X(t-\tau)$. We would like to stress that this boundary condition automatically allows us to take into account the terms proportional to $\dot{X} / c$ which lead to the appearance of the force of radiative friction (see Sec. VI).

Strictly speaking, we cannot use Lorentz transformations to change the reference frame in our case since space-time is curved. However, one can verify that corrections to formulas of Lorentz transformations due to gravitational wave are negligible.

We assume further that mirror's displacement $X(t) \sim$ $L h \ll L$. Splitting the set of equations (12, 13, 15) into the zeroth and the first order sets we obtain their solutions. It should be noted here that we have not taken into account the influence of GW on time in boundary conditions. For the zeroth order this effect vanishes since dependence on time is only included in the phase factor $e^{-i \omega_{0} t}$ which is a common multiplier for both the left and the right sides of equations. For the first order this effect is negligible since it will lead to terms of the second order in $h$. Therefore, the zeroth order solution is:

$$
\begin{aligned}
& A_{+0}=\frac{T}{1-R e^{2 i \omega_{0} \tau}} A_{\mathrm{in} 0} \\
& A_{-0}=-\frac{T e^{2 i \omega_{0} \tau}}{1-R e^{2 i \omega_{0} \tau}} A_{\mathrm{in} 0} \\
& A_{\text {out } 0}=\frac{R-e^{2 i \omega_{0} \tau}}{1-R e^{2 i \omega_{0} \tau}} A_{\mathrm{in} 0}
\end{aligned}
$$

and the first order solution in spectral domain is:

$$
\begin{aligned}
& a_{+}\left(\Omega+\omega_{0}\right)=a_{\mathrm{in}}\left(\Omega+\omega_{0}\right) \frac{T}{1-R e^{2 i\left(\Omega+\omega_{0}\right) \tau}}+\frac{R e^{2 i \omega_{0} \tau} A_{+0}}{1-R e^{2 i\left(\Omega+\omega_{0}\right) \tau} i\left[\delta \Psi_{\mathrm{mir}}(\Omega)+\delta \Psi_{\mathrm{gw}+\mathrm{emw}}(\Omega)\right],} \\
& a_{-}\left(\Omega+\omega_{0}\right)=-a_{\mathrm{in}}\left(\Omega+\omega_{0}\right) \frac{T e^{2 i\left(\Omega+\omega_{0}\right) \tau}}{1-R e^{2 i\left(\Omega+\omega_{0}\right) \tau}}+\frac{A_{-0}}{1-R e^{2 i\left(\Omega+\omega_{0}\right) \tau}} i\left[\delta \Psi_{\mathrm{mir}}(\Omega)+\delta \Psi_{\mathrm{gw}+\mathrm{emw}}(\Omega)\right],
\end{aligned}
$$




$$
a_{\text {out }}\left(\Omega+\omega_{0}\right)=a_{\text {in }}\left(\Omega+\omega_{0}\right) \frac{R-e^{2 i\left(\Omega+\omega_{0}\right) \tau}}{1-R e^{2 i\left(\Omega+\omega_{0}\right) \tau}}+\frac{T A_{-0}}{1-R e^{2 i\left(\Omega+\omega_{0}\right) \tau}} i\left[\delta \Psi_{\mathrm{mir}}(\Omega)+\delta \Psi_{\mathrm{gw}+\mathrm{emw}}(\Omega)\right] .
$$

Here we introduced the convenient notations for phase shifts after single reflection:

$$
\delta \Psi_{\operatorname{mir}}(\Omega)=2 k_{0} X(\Omega) e^{i \Omega \tau},
$$

is the phase shift acquired by the light wave when reflecting from the movable mirror. Note, that in time domain it comes with delay $t-\tau$ since it takes light wave time $\tau$ to travel from the end-mirror to the input mirror;

$$
\delta \Psi_{\mathrm{gw}+\mathrm{emw}}(\Omega)=i\left[g_{-}\left(L, \Omega+\omega_{0}\right)-g_{+}\left(L, \Omega+\omega_{0}\right)\right] e^{i \Omega \tau},
$$

is the phase shift acquired by the light wave from its direct interaction with gravitational wave. Below we calculate it explicitly and show that in long-wave approximation this phase shift vanishes.

\section{PHASE SHIFT OF LIGHT WAVE IN THE CASE OF SINGLE REFLECTION}

Let us compare our results with the ones already described in literature for the case of single reflection. To do this we ignore all resonant features of our system. First, we shall rewrite our results in spectral domain explicitly. For direct coupling of gravitational wave to light wave (19), taking into account $\omega_{\mathrm{gw}} \ll \omega_{0}$, we get:

$$
\delta \Psi_{\mathrm{gw}+\mathrm{emw}}(\Omega) \approx-k_{0} \operatorname{Lh}(\Omega)\left(1-\frac{\sin \Omega \tau}{\Omega \tau}\right) e^{i \Omega \tau} .
$$

In time domain we consider the case of a monochromatic GW with spectrum $h(\Omega)=2 \pi h_{0} \delta\left(\Omega-\omega_{\mathrm{gw}}\right)+2 \pi h_{0} \delta(\Omega+$ $\left.\omega_{\mathrm{gw}}\right)$. After simple integration of formula (20) we obtain:

$$
\begin{aligned}
\delta \Psi_{\mathrm{gw}+\mathrm{emw}}(t)= & -2 k_{0} L h_{0} \cos \omega_{\mathrm{gw}}(t-\tau) \\
& +h_{0} \frac{\omega_{0}}{\omega_{\mathrm{gw}}}\left[\sin \omega_{\mathrm{gw}} t-\sin \omega_{\mathrm{gw}}(t-2 \tau)\right] .
\end{aligned}
$$

It is straightforward to verify that in long-wave approximation $L / \lambda_{\mathrm{gw}} \ll 1$ this formula reduces to

$$
\begin{aligned}
\delta \Psi_{\mathrm{gw}+\mathrm{emw}}(t) \approx & -\frac{(2 \pi)^{2}}{3} k_{0} L h_{0}\left(\frac{L}{\lambda_{\mathrm{gw}}}\right)^{2} \cos \omega_{\mathrm{gw}} t \\
& +o\left[k_{0} L h_{0}\left(\frac{L}{\lambda_{\mathrm{gw}}}\right)^{2}\right] .
\end{aligned}
$$

Let us now turn to the full phase shift $\delta \Psi_{\mathrm{LL}}=\delta \Psi_{\text {mir }}+$ $\delta \Psi_{\mathrm{gw}+\mathrm{emw}}$. Until we find mirror law of motion we do not know how displacement $X(\Omega)$ depends on gravitational wave $h(\Omega)$. However, for single reflection (the case when we can ignore the force of light pressure) this solution has been known long before: $X(\Omega)=\frac{1}{2} L h(\Omega)$. Therefore, the full signal is

$$
\delta \Psi_{\mathrm{LL}}(\Omega)=k_{0} L h(\Omega) \frac{\sin \Omega \tau}{\Omega \tau} e^{i \Omega \tau} .
$$

In time domain for monochromatic GW we get:

$$
\delta \Psi_{\mathrm{LL}}(t)=h_{0} \frac{\omega_{0}}{\omega_{\mathrm{gw}}}\left[\sin \omega_{\mathrm{gw}} t-\sin \omega_{\mathrm{gw}}(t-2 \tau)\right] .
$$

This result coincides with the one obtained in the framework of the TT gauge in Ref. [5]:

$$
\begin{aligned}
\delta \Psi_{\mathrm{TT}} & =-\frac{\omega_{0}}{2}\left\{\int_{0}^{t-2 \tau} h\left(t^{\prime}\right) d t^{\prime}-\int_{0}^{t} h\left(t^{\prime}\right) d t^{\prime}\right\} \\
& =h_{0} \frac{\omega_{0}}{\omega_{\mathrm{gw}}}\left[\sin \omega_{\mathrm{gw}} t-\sin \omega_{\mathrm{gw}}(t-2 \tau)\right]=\delta \Psi_{\mathrm{LL}} .
\end{aligned}
$$

This coincidence confirms the results of Ref. [21]: formulas for the net phase shift obtained in the TT and the LL gauges are equivalent if only in LL gauge one takes into account the effects of localized and distributed gravitational redshifts (along with displacement of mirror). Remind that distributed redshift is the direct interaction of gravitational wave with light wave. Note that we have not considered the effect of localized redshift simply because our clocks are located at $x=0$ where $g_{00}(0, t)=-1$. For generalization one may turn to Ref. 21].

Again it is straightforward to verify that in long-wave approximation the full signal reduces to

$$
\delta \Psi_{\mathrm{LL}}(t) \approx 2 k_{0} L h_{0} \cos \omega_{\mathrm{gw}} t+O\left(k_{0} L h_{0} \frac{L}{\lambda_{\mathrm{gw}}}\right),
$$

thus leading to the rising of the term containing $\left(L / \lambda_{\mathrm{gw}}\right) h_{0}$ in the full phase shift. This was not mentioned in Ref. [5] since the delay $t-\tau$ of the reflected light wave was not taken into account in $\delta \Psi_{\text {mir }}$ there: $\delta \Psi_{\text {mir }}(t)=2 k_{0} X(t-\tau)$ but not $2 k_{0} X(t)$.

\section{MIRROR EQUATION OF MOTION}

In the absence of non-gravitational forces test mass moves along the geodesic (with respect to the input mirror of the cavity where the origin of coordinate frame is set) as dictated by geodesic equation [6, 33]. It is straightforward to verify that Christoffel coefficients are $\Gamma_{00}^{1}=-x \ddot{h} / 2 c^{2}, \Gamma_{01}^{1}=\Gamma_{11}^{1}=0$ (for the motion along the $x$-axis) in metric (2), so in non-relativistic limit we obtain the following equation of motion for a free mass:

$$
\frac{d^{2} x}{d t^{2}}-\frac{1}{2} x \ddot{h}(t)=0 .
$$


We stress here that this equation is strict for any separation between the test masses. Its solution is trivial if obtained with the method of successive approximations. By assuming $x(t)=X^{(0)}(t)+X^{(1)}(t)$ with $X^{(0)}=L$ we get $X^{(1)}=X(t)=\frac{1}{2} L h(t)$.

Generalization of Eq. (22) for the case of non-geodesic motion is the following:

$$
\frac{d^{2} x}{d t^{2}}-\frac{1}{2} x \ddot{h}(t)=\frac{1}{m} F_{x}(x, \dot{x}, t)
$$

where $F$ is the force of non-gravitational nature, the force of radiation pressure in our case, and $m$ is the test mass.

In general, the force of light pressure should be calculated in curved space-time by means of General Relativity. However in Appendix B we show that this calculation can be performed in the framework of classical flat space-time electrodynamics. We should explain this statement in detail. The rule for calculation of the light pressure force in flat space-time for any given electromagnetic wavefunction $A(x, t)$ is known [33]. Obviously, there exists a general rule for calculation of light pressure on curved space-time background. Our statement is that this general "curved space-time rule" differs from "flat space-time rule" only in negligible corrections. Therefore, in our case we can apply the "flat space time rule" to $A(x, t)$ which includes gravitational wavefunction $h$ (via direct coupling of GW to EMW) without introducing any significant error. Namely, in Appendix B we estimate the resulting inaccuracy as $\delta X_{\mathrm{GR}}^{(1)}=F_{\mathrm{GR}}^{(1)} / m \Omega^{2} \approx\left(\mathcal{E}_{\mathrm{FP}} / m c^{2}\right) L h(t) \approx 10^{-17} L h(t)$ (here $\mathcal{E}_{\mathrm{FP}}$ is the full energy of light wave inside the cavity) compared to the first order solution $X^{(1)}=\frac{1}{2} L h(t)$ of Eq. (22), assuming that $\mathcal{E}_{\mathrm{FP}}=20 \mathrm{~J}$ and $m=40 \mathrm{~kg}$ (typical parameters for Advanced LIGO project).

In classical electrodynamics 33] spatial components of the radiation pressure force are $F^{i}=\int T^{i k} d S_{k}$, where $T^{i k}$ are the components of the energy-stress tensor of electromagnetic field and $d S_{k}$ is the oriented area element. Since the light beam with cross-section $S$ is parallel to the $x$-axis and normal to the mirror surface then the $x$-component of the light pressure force is $F_{x}=T^{11} S=T_{11} S$, where $T_{11}=\left(E_{z}^{2}+H_{y}^{2}\right) / 8 \pi$, $E_{z}=-(1 / c)(\partial A / \partial t)$ and $H_{y}=-\partial A / \partial x$. Therefore, we obtain the following first order equation of motion:

$$
m\left[\frac{d^{2} X}{d t^{2}}-\frac{1}{2} L \ddot{h}(t)\right]=F_{x}^{(1)}(X, \dot{X}, t),
$$

where

$$
F_{x}^{(1)}(X, \dot{X}, t)=\frac{S}{8 \pi}\left[\frac{1}{c^{2}}\left(\frac{\partial A}{\partial t}\right)^{2}+\left(\frac{\partial A}{\partial x}\right)^{2}\right]_{x=L+X(t)}^{(1)}
$$

Index (1) on the right side of equation means that only the first order in $h$ and $X$ terms should be considered. Note that the zeroth order force of light pressure is constant, therefore, we should redefine the length $L$ in all formulas, assuming that in real experiment constant force is compensated somehow. The first order force is calculated explicitly in spectral domain in Appendix C (below we use notation $F_{x}$ instead of $F_{x}^{(1)}$ ):

$$
F_{x}(\Omega)=F_{\text {fluct }}(\Omega)+F_{\mathrm{pm}}(\Omega)+F_{\mathrm{gw}+\mathrm{emw}}(\Omega),
$$

where

$$
\begin{aligned}
& F_{\text {fluct }}(\Omega) \\
& \begin{aligned}
=\frac{S k_{0}}{\pi c}[ & \frac{T^{2} A_{\mathrm{in} 0}^{*}}{1-R e^{-2 i \omega_{0} \tau}} \frac{\left(\omega_{0}+\Omega\right) e^{i \Omega \tau}}{1-R e^{2 i\left(\omega_{0}+\Omega\right) \tau}} a_{\mathrm{in}}\left(\omega_{0}+\Omega\right) \\
& \left.+\frac{T^{2} A_{\mathrm{in} 0}}{1-R e^{2 i \omega_{0} \tau}} \frac{\left(\omega_{0}-\Omega\right) e^{i \Omega \tau}}{1-R e^{-2 i\left(\omega_{0}-\Omega\right) \tau}} a_{\mathrm{in}}^{*}\left(\omega_{0}-\Omega\right)\right],
\end{aligned}
\end{aligned}
$$

is the fluctuation part of the force (see Appendix C2), which leads to the quantum radiation pressure noise (back-action) after the quantization of the light field;

$$
\begin{aligned}
F_{\mathrm{pm}}(\Omega) & =-\mathcal{K}(\Omega) X(\Omega) \\
& =-K(\Omega) X(\Omega)+2 i \Omega \Gamma(\Omega) X(\Omega),
\end{aligned}
$$

is the ponderomotive force, where (see Appendix C3):

$$
K(\Omega)=\frac{4 k_{0} S W_{\mathrm{FP}} R e^{2 i \Omega \tau} \sin 2 \omega_{0} \tau}{1-2 R e^{2 i \Omega \tau} \cos 2 \omega_{0} \tau+R^{2} e^{4 i \Omega \tau}},
$$

is the coefficient of optical rigidity and

$$
\Gamma(\Omega)=\frac{S W_{\mathrm{FP}}}{c} \frac{1-R^{2} e^{4 i \Omega \tau}}{1-2 R e^{2 i \Omega \tau} \cos 2 \omega_{0} \tau+R^{2} e^{4 i \Omega \tau}},
$$

is the coefficient of radiative friction. Here $W_{\mathrm{FP}}=$ $k_{0}^{2}\left(A_{+0} A_{+0}^{*}+A_{-0} A_{-0}^{*}\right) / 2 \pi$ is the energy density of light wave circulating inside the cavity. Formula (24) was obtained in Refs. [11, 12, 18] and formula (25) in Ref. [18], where phenomenon of radiative friction was discussed in detail.

We split up $\mathcal{K}$ into $K$ and $2 i \Omega \Gamma$ using the following reasoning. The obtained mechanical impedance $Z(\Omega)$ of the mirror is $Z(\Omega)=m \Omega^{2}+2 i \Omega \Gamma(\Omega)-K(\Omega)$ which obviously looks similar to the impedance of linear harmonic oscillator. Therefore, $\mathcal{K}(\Omega)$ includes the term $2 i \Omega \Gamma(\Omega)$ describing friction, and the term $K(\Omega)$ describing rigidity, where $2 i \Omega \Gamma$ is of the order of $\left(\Omega / \omega_{0}\right) K$. Radiative friction $\Gamma(\Omega)$ appears due to the boundary condition (14) which takes into account the terms proportional to $\dot{X} / c$. This relativistic correction was not considered in Ref. [11].

Both the terms have real and imaginary parts. The latter means that both effects are introduced with delay. Note that the coefficient of "true" friction is only the real part of formula (25). "True" friction is always masked with the imaginary part of formula (24) since $|K| \gg|2 \Omega \Gamma|$, except for the case of optical resonance (see later) when optical rigidity vanishes. In non-resonant regime there exist two possibilities (in long-wave approximation), depending on the sign of $\sin 2 \omega_{0} \tau$ : either (i) 
$\mathfrak{R}[K(\Omega)]>0$ (positive rigidity) and $\mathfrak{I}[K(\Omega)]<0$ (negative damping), or (ii) $\mathfrak{R}[K(\Omega)]<0$ (negative rigidity) and $\mathfrak{I}[K(\Omega)]>0$ (positive damping). These inequalities were first obtained in Refs. [7, 8].

The concept of ponderomotive rigidity and the role it plays in standard quantum limited (SQL) detectors has been widely discussed in literature before (for longwave approximation only). For the first time it appeared in Refs. [7, 8] where microwave resonators were considered. Optical ponderomotive rigidity and its application to large-scale interferometric GW detectors were studied in Refs. [9, 10, 11, 12]. Papers [13, 14, 15, 16, 17] dealt with the optical springs in signal-recycling topologies of LIGO detectors;

$$
F_{\text {gw }+\mathrm{emw}}(\Omega)=\frac{1}{2} \mathcal{K}(\Omega)\left(1-\frac{\sin \Omega \tau}{\Omega \tau}\right) \operatorname{Lh}(\Omega),
$$

is the 'GW+EMW' correction to the force of radiation pressure (see Appendix C4), arising when the cavity length becomes comparable with gravitational wavelength. Note, that it is proportional to $\delta \Psi_{\mathrm{gw}+\mathrm{emw}}(\Omega)$ much as ponderomotive force is proportional to $\delta \Psi_{\text {mir }}(\Omega)$. Obviously, in long-wave approximation $L \ll \lambda_{\mathrm{gw}}$ or $\Omega \tau \ll 1$ this 'GW+EMW' correction vanishes (along with the corresponding phase shift). Similar formula (without $\Gamma$ in $\mathcal{K}$ ) was obtained in Ref. [11].

\section{MIRROR'S LAW OF MOTION AND DETECTOR'S RESPONSE SIGNAL}

Now we solve Eq. (23) in spectral domain:

$$
X(\Omega)=\frac{1}{2} \frac{m \Omega^{2} L h(\Omega)}{m \Omega^{2}-\mathcal{K}(\Omega)}-\frac{F_{\text {gw }+\mathrm{emw}}(\Omega)+F_{\text {fluct }}(\Omega)}{m \Omega^{2}-\mathcal{K}(\Omega)} .
$$

In this paper we are mostly interested in dynamical part of the problem, therefore, we shall ignore fluctuations (terms containing $a_{\text {in }}$ and $F_{\text {fluct }}$ ) further. Substituting (26) into (27) we obtain:

$$
X(\Omega)=\frac{1}{2} \frac{L h(\Omega)}{m \Omega^{2}-\mathcal{K}(\Omega)}\left[m \Omega^{2}-\mathcal{K}(\Omega)\left(1-\frac{\sin \Omega \tau}{\Omega \tau}\right)\right] .
$$

Strictly speaking, in order to keep all the $\dot{X} / c$ proportional terms we should expand $g_{ \pm}$in formula (19) up to the first order of $\Omega / \omega_{0}$ and then replace $(1-\operatorname{sinc} \Omega \tau)$ with $\left(1-\Omega / \omega_{0}\right)(1-\operatorname{sinc} \Omega \tau)$ in formulas (26) and (28). However, for $\Omega / 2 \pi \sim 10^{2} \div 10^{5} \mathrm{~Hz}$ we have $\Omega / \omega_{0} \sim 10^{-12} \div 10^{-9}$ and this correction can be omitted in both the cases $K(\Omega) \neq 0$ (term $K \times \Omega / \omega_{0}$ is negligible) and $K(\Omega)=0\left(\right.$ term $2 \Omega \Gamma \times \Omega / \omega_{0} \propto \dot{X}^{2} / c^{2}$ is negligible). In addition, the term in square brackets in formula (28) does not contain corrections of the order of $\left(\mathcal{E}_{\mathrm{FP}} / m c^{2}\right) m \Omega^{2} \approx 10^{-17} m \Omega^{2}$ since we have omitted them when calculating the radiation pressure force.
Substituting formulas (20) and (28) into (17c) we finally obtain the explicit formula for the optical field of the signal wave:

$$
\begin{aligned}
a_{\text {out }}\left(\Omega+\omega_{0}\right)= & -A_{\text {in } 0} \frac{T^{2} e^{2 i \omega_{0} \tau}}{\left(1-R e^{2 i \omega_{0} \tau}\right)\left(1-R e^{2 i\left(\Omega+\omega_{0}\right) \tau}\right)} \\
& \times \frac{m \Omega^{2}}{m \Omega^{2}-\mathcal{K}(\Omega)} i k_{0} \operatorname{Lh}(\Omega) \frac{\sin \Omega \tau}{\Omega \tau} e^{i \Omega \tau} .
\end{aligned}
$$

Similarly to the previous formula one can replace $\operatorname{sinc} \Omega \tau$ with $\left[1-\left(1-\Omega / \omega_{0}\right)(1-\operatorname{sinc} \Omega \tau)\right]$ for strictness, but for the reasons discussed above this correction is negligible.

Note that the obtained response signal is the product of three terms: optical resonant multiplier, mechanical resonant multiplier and the term (21) describing the single reflection response. The latter is proportional to sincfunction and is sometimes referred to as the "electric" component of the detector full (single reflection) angular pattern [35]. Recently it was demonstrated that the angular pattern also contains the so-called "magnetic" component [35] (due to non-optimal GW polarization and detector orientation in general case) with its amplitude proportional to $\Omega \tau$ in low frequencies but rising with increasing frequency [36]. Therefore, it may be interesting to perform a (straightforward) generalization of our results for the case of generic GW polarization and direction of propagation. Probably, it will add up to the multiplication of the known single reflection angular pattern by the same optical and mechanical resonant multipliers as in formula (29) but, strictly speaking, this problem requires a detailed analysis.

In many cases the phenomenon of radiative friction is negligible in comparison with optical rigidity since $|2 \Omega \Gamma| \ll K$. Then one should replace $\mathcal{K}(\Omega) \rightarrow K(\Omega)$ in formulas (28) and (29).

One of the most interesting effects which may arise when detecting the high-frequency GW signal is parametric transitions between optical modes of the cavity if $\Omega=\omega_{\mathrm{gw}} \approx n \omega_{\mathrm{fsr}}$, where $n \in \mathbb{N}$ and $\omega_{\mathrm{fsr}}=\pi c / L$ is the cavity free spectral range (see Fig. 3). In some sense this effect looks similar to the phenomenon of parametric oscillatory instability (POI) described in Refs. [37, 38]. The difference is in the following: POI appears due to the coupling of optical modes to acoustic (mechanical) modes of the movable mirror while the effect illustrated in Fig. 3 appears due to the coupling of optical modes to gravitational wave itself. This effect can be used for detection of high-frequency signals and requires additional analysis.

\section{SPECIAL CASES}

It is useful to examine the obtained results in two special cases - optical resonance and small detuning from it - and compare our results with the ones already present in literature. We shall use notations for the energy of 


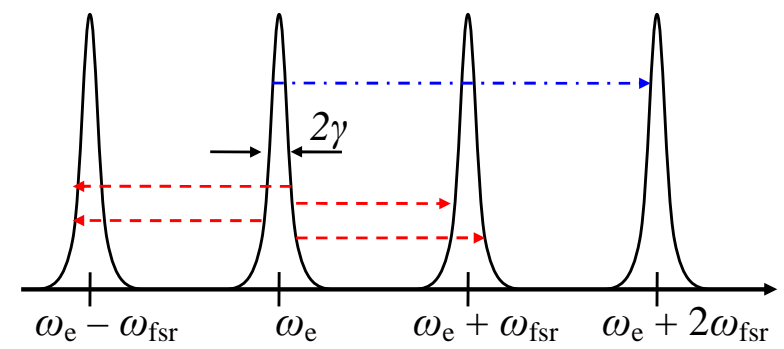

FIG. 3: Optical resonances of Fabry-Perot cavity: $\omega_{\mathrm{e}}$ is one of the eigenfrequencies, $\omega_{\mathrm{fsr}}=\pi c / L$ is the cavity free spectral range, $\gamma=(1-R) / 2 \tau$ is cavity half-bandwidth. Red dashed arrows denote some possible transitions between neighboring resonant curves under the influence of $\mathrm{GW}$ with frequency equal or approximately equal to FSR: $\omega_{\mathrm{gw}} \approx \omega_{\mathrm{fsr}}$. Blue dashdotted line denote one of the possible transitions between nonneighboring curves if $\omega_{\mathrm{gw}} \approx 2 \omega_{\mathrm{fsr}}$.

light wave inside the cavity $\mathcal{E}_{\mathrm{FP}}=W_{\mathrm{FP}} S L$, and cavity half-bandwidth $\gamma=(1-R) / 2 \tau$. In these notations $T^{2}=1-R^{2} \approx 4 \gamma \tau$ and $\mathcal{E}_{\mathrm{FP}}=2 P_{\text {in }} / \gamma$, where $P_{\text {in }}$ is the input power.

\section{A. Optical resonance}

Let us assume that frequency of the pump wave $\omega_{0}$ coincides with one of the eigenfrequencies of a Fabry-Perot cavity: $\omega_{0} \tau=\pi n, n \in \mathbb{N}$. We shall denote all quantities with index 'res' in this section. Then, simplifying formulas (24), (25), (28) and (29) we obtain

$$
K^{\mathrm{res}}(\Omega)=0, \quad \Gamma^{\mathrm{res}}(\Omega)=\frac{S W_{\mathrm{FP}}}{c} \frac{1+R e^{2 i \Omega \tau}}{1-R e^{2 i \Omega \tau}},
$$

thus in the case of resonance rigidity vanishes and radiative friction is not masked by it;

$$
\begin{aligned}
X^{\mathrm{res}}(\Omega)=\frac{1}{2} & L h(\Omega)\left[1-\frac{2 i \Omega \Gamma^{\mathrm{res}}(\Omega)}{m \Omega^{2}+2 i \Omega \Gamma^{\mathrm{res}}(\Omega)} \frac{\sin \Omega \tau}{\Omega \tau}\right], \\
a_{\mathrm{out}}^{\mathrm{res}}\left(\Omega+\omega_{0}\right)= & -A_{\mathrm{in} 0} \frac{2}{1-R e^{2 i \Omega \tau}} \frac{m \Omega^{2}}{m \Omega^{2}+2 i \Omega \Gamma^{\mathrm{res}}(\Omega)} \\
& \times i k_{0} L h(\Omega) \frac{\sin \Omega \tau}{\Omega \tau} e^{i \Omega \tau} .
\end{aligned}
$$

However, radiative friction is still a tiny effect even in the case of optical resonance and is omitted usually:

$$
\begin{aligned}
X^{\mathrm{res}}(\Omega) & \approx \frac{1}{2} \operatorname{Lh}(\Omega), \\
a_{\mathrm{out}}^{\mathrm{res}}\left(\Omega+\omega_{0}\right) & \approx-A_{\text {in } 0} \frac{2}{1-R e^{2 i \Omega \tau}} i k_{0} L h(\Omega) \frac{\sin \Omega \tau}{\Omega \tau} e^{i \Omega \tau} .
\end{aligned}
$$

Therefore, in the case of optical resonance light field inside the cavity does not act dynamically on test mass if we neglect radiative friction, i.e. test mass is inertial.
Resonant features of the cavity only appear in frequencydependent amplification of the light wave amplitude.

In long-wave approximation these formulas, denoted with 'lw', reduce to the well-known ones:

$$
K_{\mathrm{lw}}^{\mathrm{res}}(\Omega)=0, \quad \Gamma_{\mathrm{lw}}^{\mathrm{res}}(\Omega) \approx \frac{\mathcal{E}_{\mathrm{FP}}}{L^{2}} \frac{1+i \Omega \tau}{\gamma-i \Omega} .
$$

The coefficient of "true" friction is the real part of $\Gamma_{\mathrm{lw}}^{\mathrm{res}}$ :

$$
\mathfrak{R}\left[\Gamma_{\mathrm{lw}}^{\mathrm{res}}(\Omega)\right]=\frac{\mathcal{E}_{\mathrm{FP}}}{L^{2}} \frac{\gamma}{\gamma^{2}+\Omega^{2}}=\frac{32 P_{\mathrm{in}}}{c^{2}} \frac{1}{T^{4}+(4 \Omega \tau)^{2}} .
$$

Last formula with $P_{\text {in }}$ coincides with the one in Ref. [18];

$$
\begin{aligned}
& X_{\mathrm{lw}}^{\mathrm{res}}(\Omega)=\frac{1}{2} \operatorname{Lh}(\Omega) \frac{m \Omega^{2}}{m \Omega^{2}+2 i \Omega \Gamma_{\mathrm{lw}}^{\mathrm{res}}(\Omega)} \approx \frac{1}{2} \operatorname{Lh}(\Omega), \\
& a_{\mathrm{out}, \mathrm{lw}}^{\mathrm{res}}\left(\Omega+\omega_{0}\right) \\
& \quad \approx-A_{\mathrm{in} 0} \frac{1}{(\gamma-i \Omega) \tau} \frac{m \Omega^{2}}{m \Omega^{2}+2 i \Omega \Gamma_{\mathrm{lw}}^{\mathrm{res}}(\Omega)} i k_{0} L h(\Omega) \\
& \quad \approx-A_{\mathrm{in} 0} \frac{1}{(\gamma-i \Omega) \tau} i k_{0} \operatorname{Lh}(\Omega) .
\end{aligned}
$$

\section{B. Small detuning from optical resonance}

We shall now turn to the case when frequency of the pump wave is slightly shifted from one of the eigenfrequencies:

$$
\omega_{0}=\frac{\pi n}{\tau}+\delta, \quad \delta \tau \ll 1 .
$$

Again, simplifying formulas (24), (28) and (29) we obtain (all quantities in this section are marked with 'det'):

$$
K^{\operatorname{det}}(\Omega)=\frac{8 k_{0} S W_{\mathrm{FP}} R e^{2 i \Omega \tau} \delta \tau}{\left(1-R e^{2 i \Omega \tau}\right)^{2}+4 R(\delta \tau)^{2} e^{2 i \Omega \tau}} .
$$

The graphs of $\mathfrak{R}\left[K^{\operatorname{det}}(\Omega)\right]$ ("true" rigidity) and $\mathfrak{I}\left[K^{\operatorname{det}}(\Omega)\right]$ (damping) are plotted in Fig. (4). We do not consider radiative friction here since $|K(\Omega)| \gg|2 \Omega \Gamma(\Omega)|$;

$$
\begin{aligned}
X^{\operatorname{det}}(\Omega)= & \frac{1}{2} L h(\Omega)\left[1+\frac{K^{\operatorname{det}}(\Omega)}{m \Omega^{2}-K^{\operatorname{det}}(\Omega)} \frac{\sin \Omega \tau}{\Omega \tau}\right], \\
a_{\text {out }}^{\operatorname{det}}\left(\Omega+\omega_{0}\right)= & -A_{\text {in } 0} \frac{2 \gamma}{\gamma-i \delta} \frac{1+2 i \delta \tau}{1-R(1+2 i \delta \tau) e^{2 i \Omega \tau}} \\
& \times \frac{m \Omega^{2}}{m \Omega^{2}-K^{\operatorname{det}}(\Omega)} i k_{0} L h(\Omega) \frac{\sin \Omega \tau}{\Omega \tau} e^{i \Omega \tau} .
\end{aligned}
$$

Therefore, optical rigidity appears only when pumping frequency is detuned from resonance.

In long-wave approximation ('lw') we obtain:

$$
K_{\mathrm{lw}}^{\mathrm{det}}(\Omega)=\frac{2 \omega_{0} \mathcal{E}_{\mathrm{FP}}}{L^{2}} \frac{\delta}{\delta^{2}+(\gamma-i \Omega)^{2}},
$$



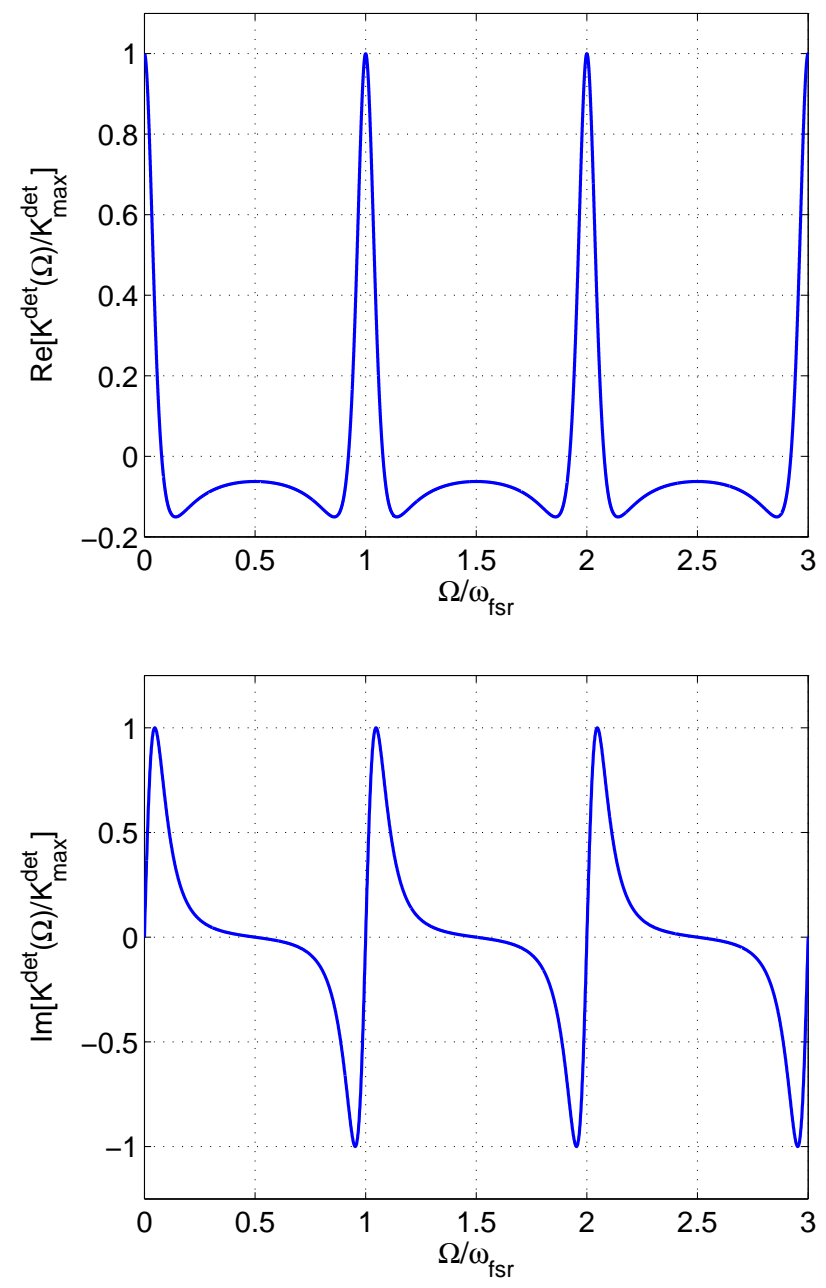

FIG. 4: "True" rigidity (upper figure) and damping (lower figure) normalized to their maximum values. For visualization we assumed $R=0.6, \delta / 2 \pi=100 \mathrm{~Hz}$.

coinciding with the corresponding formula in Ref. [12]. One can easily check that the ratio $\mathfrak{R}\left[K^{\operatorname{det}}(\Omega)\right] /\left(m \Omega^{2}\right)$ can be made of the order of unity for the values of parameters planned for Advanced LIGO $\left(L=4 \mathrm{~km}, \mathcal{E}_{\mathrm{FP}} \leq 20\right.$ $\mathrm{J}, m=40 \mathrm{~kg}, \omega_{0} / 2 \pi=3 \times 10^{14} \mathrm{~Hz}, \gamma / 2 \pi \approx 1 \mathrm{~Hz}$, $\Omega / 2 \pi \approx \delta / 2 \pi=100 \mathrm{~Hz})$;

$$
\begin{aligned}
X_{\mathrm{lw}}^{\mathrm{det}}(\Omega) \approx & \frac{m \Omega^{2}}{m \Omega^{2}-K_{\mathrm{lw}}^{\operatorname{det}}(\Omega)} \frac{1}{2} \operatorname{Lh}(\Omega), \\
a_{\mathrm{out}, \mathrm{lw}}^{\mathrm{det}}\left(\Omega+\omega_{0}\right) \approx & -A_{\mathrm{in} 0} \frac{\gamma / \tau}{\gamma-i \delta} \frac{1+2 i \delta \tau}{\gamma-i(\delta+\Omega)} \\
& \times \frac{m \Omega^{2}}{m \Omega^{2}-K_{\mathrm{lw}}^{\operatorname{det}}(\Omega)} i k_{0} L h(\Omega) .
\end{aligned}
$$

Formulas (32 34) are applicable to consideration of the signal-recycling topology since it is equivalent (with some modifications of parameters) to a single detuned FabryPerot cavity [15]. Therefore, all the major results of Refs.
[13, 14, 16, 17] which studied in detail optical springs in SR topologies hold true for a single cavity.

\section{Response function of the detuned cavity in the vicinity of FSR}

To analyze the operation of GW detector near the FSR we turn to formulas (30) and (31). Introducing notation $\Delta \equiv \Omega-\omega_{\mathrm{fsr}},|\Delta| \ll \omega_{\mathrm{fsr}}$ we rewrite these formulas in the following way:

$$
K^{\operatorname{det}}(\Delta)=\frac{2 \omega_{0} \mathcal{E}_{\mathrm{FP}}}{L^{2}} \frac{\delta}{\delta^{2}+(\gamma-i \Delta)^{2}} .
$$

We plotted $\mathfrak{R}\left[K^{\operatorname{det}}(\Delta)\right]$ and $\mathfrak{I}\left[K^{\operatorname{det}}(\Delta)\right]$ in the vicinity of $\Delta=0$ (or, equivalently, near $\Omega=\omega_{\mathrm{fsr}}$ ) in Fig. 5. Note that while decreasing the cavity half-bandwidth from $\gamma \sim \delta$ to $\gamma \ll \delta$ the shape of $\mathfrak{R}\left[K^{\operatorname{det}}(\Delta)\right]$ changes from "singlet" to "doublet". Obviously, this takes place in the vicinity of each $\Omega=n \omega_{\mathrm{fsr}}, n \in \mathbb{Z}$;

$$
\begin{aligned}
& a_{\mathrm{out}}^{\mathrm{det}}\left(\omega_{\mathrm{fsr}}+\Delta+\omega_{0}\right) \\
& \approx- \\
& \quad-A_{\mathrm{in} 0} \frac{\gamma / \tau}{\gamma-i \delta} \frac{1+2 i \delta \tau}{\gamma-i(\delta+\Delta)} \\
& \quad \times \frac{m \omega_{\mathrm{fsr}}^{2}}{m \omega_{\mathrm{fsr}}^{2}-K^{\operatorname{det}}(\Delta)} \frac{\Delta}{\omega_{\mathrm{fsr}}} i k_{0} \operatorname{Lh}\left(\omega_{\mathrm{fsr}}+\Delta\right) .
\end{aligned}
$$

The ratio $\mathfrak{R}\left[K^{\operatorname{det}}(\Delta)\right] /\left(m \omega_{\text {fsr }}^{2}\right)$ can be estimated as $\approx$ $10^{-4}$ (see Fig. 6) for $L=4 \mathrm{~km}, \omega_{0} / 2 \pi=3 \times 10^{14} \mathrm{~Hz}$, $\delta / 2 \pi=100 \mathrm{~Hz}, \gamma / 2 \pi=1 \mathrm{~Hz}, \mathcal{E}_{\mathrm{FP}}=20 \mathrm{~J}, m=40$ $\mathrm{kg}$ and $\omega_{\mathrm{fsr}} / 2 \pi=37.5 \mathrm{kHz}$. Thus the implementation of optical rigidity for the detection of signals near the FSR will require the increase of circulating optical power, the decrease of cavity half-bandwidth (see Fig. 6) and the mirror mass. With these requirements met it will be possible to perform the resonant detection of highfrequency gravitational waves in the vicinity of $37.5 \mathrm{kHz}$. Note that while $m \omega_{\mathrm{fsr}}^{2}-\mathfrak{R}\left[K^{\operatorname{det}}(\Delta)\right]$ can be made vanish for certain frequencies in principle, the resonant gain will still be limited by $m \omega_{\mathrm{fsr}}^{2} / \Im\left[K^{\operatorname{det}}(\Delta)\right]$.

\section{CONCLUSION}

In this paper we have analyzed the interaction of ' + 'polarized plane gravitational waves with a Fabry-Perot cavity. Calculations have been performed in its input mirror's local Lorentz frame which is best suited for consideration of non-gravitational forces acting on test masses, such as the force of radiation pressure. We have considered the general case of ratio $L / \lambda_{\text {gw }}$ ( $L$ is the cavity length) for the purpose of high-frequency GW signals detection when long-wave approximation is violated. Such high-frequency gravitational waves can be emitted by the sources predicted in modern astrophysical and cosmological theories, for example, relic GW background. 

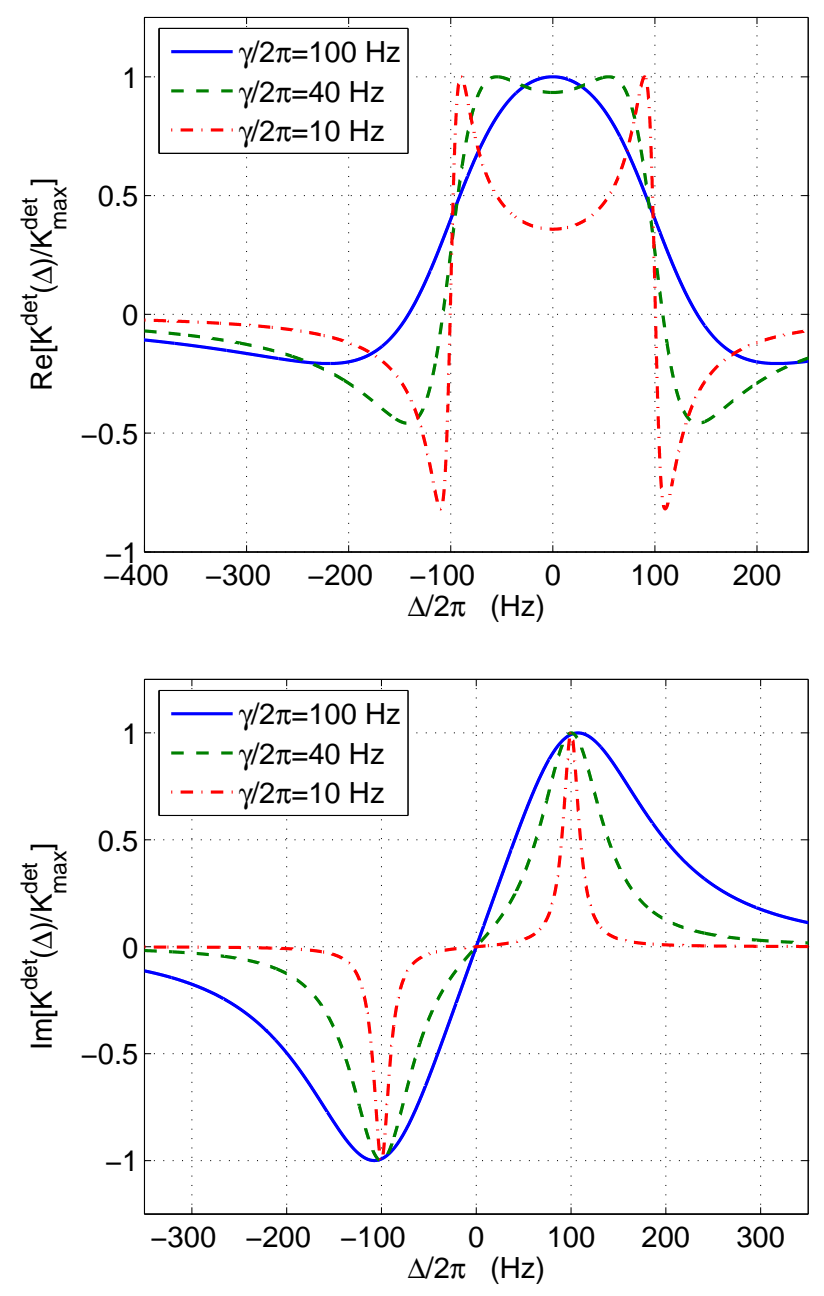

FIG. 5: Normalized rigidity (upper figure) and damping (lower figure) as functions of detuning from the FSR. For visualization we assumed $\delta / 2 \pi=100 \mathrm{~Hz}$.

We began from the solution of wave equation for running light wave which is coupled to a weak plane gravitational wave and found that in long-wave approximation this interaction leads to a small correction of the order of $\left(x / \lambda_{\mathrm{gw}}\right)^{2} h$, where $x$ is the distance traveled by light, in agreement with Ref. [5]. However if $x \sim \lambda_{\mathrm{gw}}$ direct interaction between gravitational wave and light wave becomes significant and is described by oscillating function. We applied this result to consideration of the boundary problem for the light wave in a Fabry-Perot cavity with the movable mirror. It was shown that the response signal of such GW detector includes two terms: (i) the phase shift of light due to reflection from the movable mirror and (ii) the phase shift due to direct coupling of gravitational wave to light wave. In our analysis we assumed that the end-mirror of the cavity moves under the influence of (i) gravitational wave and (ii) the force of light pressure, neglecting all other forces. As a special
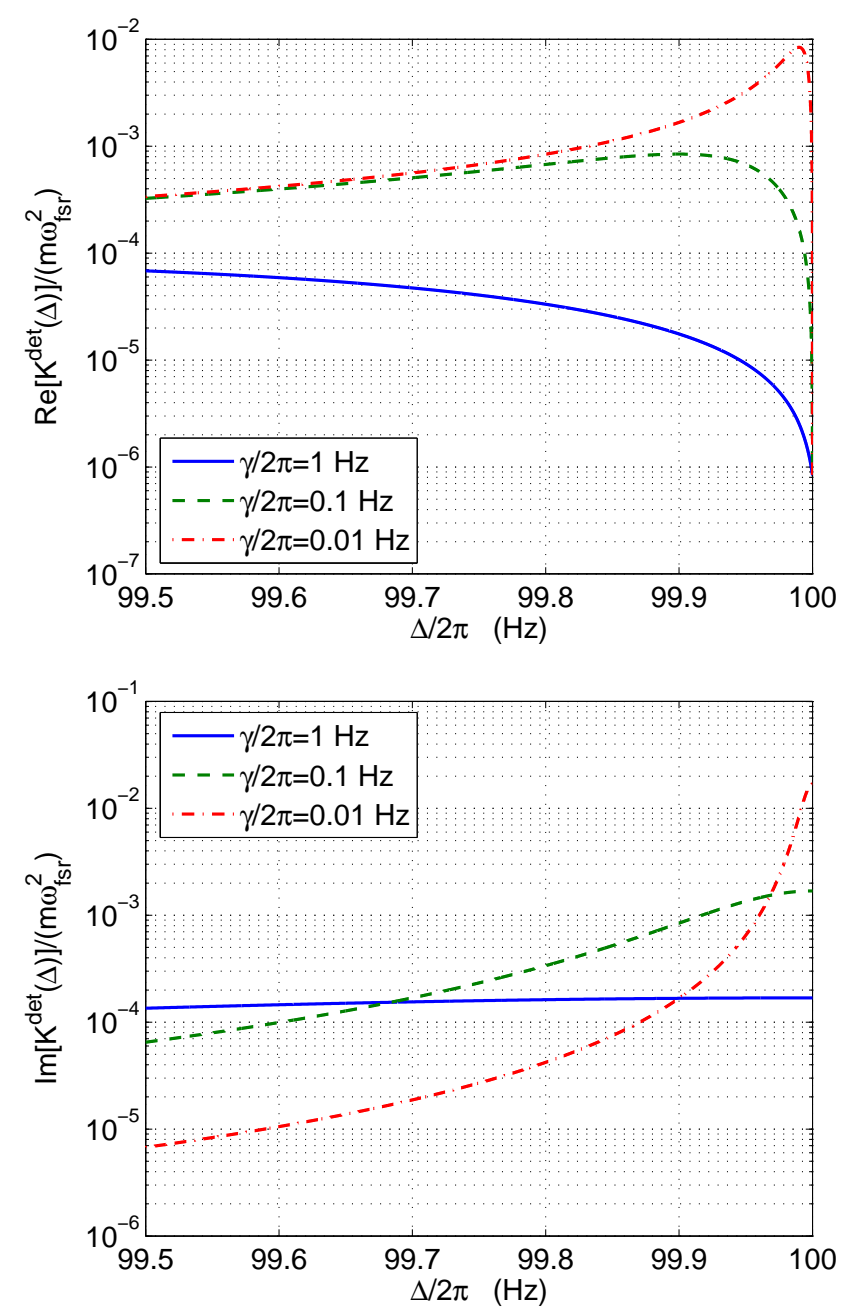

FIG. 6: Rigidity (upper figure) and damping (lower figure) near the FSR compared to $m \omega_{\mathrm{fsr}}^{2}$. For visualization we assumed $L=4 \mathrm{~km}, \omega_{0} / 2 \pi=3 \times 10^{14} \mathrm{~Hz}, \delta / 2 \pi=100 \mathrm{~Hz}$, $\mathcal{E}_{\mathrm{FP}}=20 \mathrm{~J}, m=40 \mathrm{~kg}$ and $\omega_{\mathrm{fsr}} / 2 \pi=37.5 \mathrm{kHz}$.

case, by neglecting the resonant features of the cavity, we examined the situation of single reflection when radiation pressure is negligible. In this case test mass undergoes geodesic motion only and, therefore, is inertial. TT gauge can be applied then to the analysis of GW detector. This was performed in Ref. [5] and we found that our LL-based results coincide with the TT-based results. This also confirmed the results of Ref. [21]: in order to get the agreement between the LL- and the TT-results direct interaction of the gravitational wave with the light wave must be taken into account in the LL gauge along with the displacement of test masses.

Next we considered the mirror equation of nongeodesic motion in the field of gravitational wave and the force of light pressure. Traditionally in literature this is done only in long-wave approximation. We proposed rather general method of calculation of radiation 
pressure when condition $L \ll \lambda_{\text {gw }}$ is violated and showed that this calculation can be performed in flat space-time without introducing a significant error. We obtained the formulas for coefficients of ponderomotive optical rigidity and radiative friction along with the general relativistic correction describing the influence of direct 'GW+EMW' interaction on the force of radiation pressure. This correction must be taken into account in order to get correct formula for the response signal when long-wave approximation is violated. Though formulas for optical rigidity, radiative friction and 'GW+EMW' correction separately have been presented in literature before [11, 12, 18], nobody have obtained them simultaneously using one unified method, as we have done in this paper. The phenomenon of optical rigidity plays significant role in operation of proposed schemes for advanced GW detectors and our results may be of potential interest in consideration of the regimes of high-frequency GW signals detection. One may also apply our results to calculate the modification of the detector angular pattern, including its magnetic component $[35,36]$, due to radiation pressure effects.

The possibility of parametric transitions between different resonant curves of the cavity under the influence of gravitational waves with frequencies $\omega_{\mathrm{gw}} \approx n \omega_{\mathrm{fsr}}$ was discussed. This effect may find implementation in detection of the high-frequency GW signals using optical modes with frequencies $\omega_{0} \pm n \omega_{\mathrm{fsr}}$ as signal waves instead of the $\omega_{0}$-input mode.

In the end we considered two special cases of optical resonance and small detuning from it. Excellent agreement with results of [12, 18] was found. We also estimated the deposit of optical rigidity into the response signal near the FSR and showed that in order to perform the resonant detection of GWs near that frequency one should increase the circulating optical power and decrease cavity half-bandwidth and the end-mirror mass.

\section{Acknowledgments}

I would like to thank S.P. Vyatchanin and F.Ya. Khalili for useful comments on the paper and fruitful discussions on physics of interferometric GW antennas; V.I. Denisov for his comments and advice on general relativistic aspects of my work; S.L. Danilishin for careful reading of the manuscript and correction of errors in the text. I also would like to express the sincerest gratitude to Malik Rakhmanov who devoted much time and effort to give me detailed comments and advice on the paper, pointed out my inaccuracies and uncertainties and gave me some references I was not aware of. I also thank him for very interesting and fruitful discussion on highfrequency GWs and future prospects of their detection.

This work was supported by LIGO team from Caltech and in part by NSF and Caltech grant PHY-0353775, by the Russian Agency of Industry and Science, contracts No. 5178.2006.2 and 02.445.11.7423. This article has been assigned LIGO doc. number LIGO-P060055-01-R. 


\section{APPENDIX A: SOLUTION OF THE FIRST ORDER EQUATION FOR RUNNING EM WAVE}

In this Appendix we obtain the solution of the Cauchy problem (5-7).

It is convenient to go to spectral domain. Applying the theorem of convolution to the right side of Eq. (5), we get the following first-order equation in spectral domain:

$$
\begin{aligned}
\frac{\partial^{2} A^{(1)}}{\partial x^{2}}+\frac{\Omega^{2}}{c^{2}} & A^{(1)} \\
=\frac{1}{2} k_{0}^{2} x^{2} & {\left[A_{+0} e^{i k_{0} x} \frac{\left(\Omega-\omega_{0}\right)^{2}}{c^{2}} h\left(\Omega-\omega_{0}\right)\right.} \\
& +A_{+0}^{*} e^{-i k_{0} x} \frac{\left(\Omega+\omega_{0}\right)^{2}}{c^{2}} h\left(\Omega+\omega_{0}\right) \\
& +A_{-0} e^{-i k_{0} x} \frac{\left(\Omega-\omega_{0}\right)^{2}}{c^{2}} h\left(\Omega-\omega_{0}\right) \\
& \left.+A_{-0}^{*} e^{i k_{0} x} \frac{\left(\Omega+\omega_{0}\right)^{2}}{c^{2}} h\left(\Omega+\omega_{0}\right)\right]
\end{aligned}
$$

where

$$
\begin{aligned}
& A^{(1)}(x, \Omega)=A_{+}^{(1)}(x, \Omega)+A_{-}^{(1)}(x, \Omega), \\
& A_{+}^{(1)}(0, \Omega)=A_{-}^{(1)}(0, \Omega)=0 .
\end{aligned}
$$

are equivalent to formulas (6) and (7) correspondingly. For convenience we introduce the following notations:

$$
\begin{aligned}
& A_{+}^{(1)}(x, \Omega) \equiv A, \quad k \equiv \frac{\Omega}{c}, \\
& \mathcal{B}_{1} \equiv \frac{1}{2} k_{0}^{2} A_{+0} \frac{\left(\Omega-\omega_{0}\right)^{2}}{c^{2}} h\left(\Omega-\omega_{0}\right) \\
& \mathcal{B}_{2} \equiv \frac{1}{2} k_{0}^{2} A_{+0}^{*} \frac{\left(\Omega+\omega_{0}\right)^{2}}{c^{2}} h\left(\Omega+\omega_{0}\right) \\
& \mathcal{B}_{3} \equiv \frac{1}{2} k_{0}^{2} A_{-0} \frac{\left(\Omega-\omega_{0}\right)^{2}}{c^{2}} h\left(\Omega-\omega_{0}\right) \\
& \mathcal{B}_{4} \equiv \frac{1}{2} k_{0}^{2} A_{-0}^{*} \frac{\left(\Omega+\omega_{0}\right)^{2}}{c^{2}} h\left(\Omega+\omega_{0}\right) .
\end{aligned}
$$

Eq. (A1a) rewritten in these notations is:

$$
\frac{d^{2} A}{d x^{2}}+k^{2} A=\left(\mathcal{B}_{1}+\mathcal{B}_{4}\right) x^{2} e^{i k_{0} x}+\left(\mathcal{B}_{2}+\mathcal{B}_{3}\right) x^{2} e^{-i k_{0} x} .
$$

Note that spectral frequency $\Omega$ (or $k=\Omega / c$ ) plays the role of parameter here. General solution of homogeneous equation $d^{2} A / d x^{2}+k^{2} A=0$ is well-known: $A(x)=$ $C_{1} e^{i k x}+C_{2} e^{-i k x}$. We shall obtain the particular solution of heterogeneous Eq. (A2) using the method of variation of constants (see any ODE handbook), assuming

$$
A(x)=C_{1}(x) e^{i k x}+C_{2}(x) e^{-i k x} .
$$

Following the general rule of this method we must solve the following set of equations:

$$
\frac{d C_{1}}{d x} e^{i k x}+\frac{d C_{2}}{d x} e^{-i k x}=0,
$$

$$
\begin{aligned}
i k \frac{d C_{1}}{d x} e^{i k x}-i k \frac{d C_{2}}{d x} e^{-i k x}= & \left(\mathcal{B}_{1}+\mathcal{B}_{4}\right) x^{2} e^{i k_{0} x} \\
& +\left(\mathcal{B}_{2}+\mathcal{B}_{3}\right) x^{2} e^{-i k_{0} x} .
\end{aligned}
$$

It is straightforward to verify that solution is

$$
\begin{aligned}
C_{1}(x)= & \frac{\mathcal{B}_{1}+\mathcal{B}_{4}}{2 i k} e^{i\left(k_{0}-k\right) x} \\
& \times\left(\frac{x^{2}}{i\left(k_{0}-k\right)}+\frac{2 x}{\left(k_{0}-k\right)^{2}}-\frac{2}{i\left(k_{0}-k\right)^{3}}\right) \\
+ & \frac{\mathcal{B}_{2}+\mathcal{B}_{3}}{2 i k} e^{-i\left(k_{0}+k\right) x} \\
& \times\left(-\frac{x^{2}}{i\left(k_{0}+k\right)}+\frac{2 x}{\left(k_{0}+k\right)^{2}}+\frac{2}{i\left(k_{0}+k\right)^{3}}\right) \\
+ & C_{10} .
\end{aligned}
$$

and

$$
\begin{aligned}
C_{2}(x)= & -\frac{\mathcal{B}_{1}+\mathcal{B}_{4}}{2 i k} e^{i\left(k_{0}+k\right) x} \\
& \times\left(\frac{x^{2}}{i\left(k_{0}+k\right)}+\frac{2 x}{\left(k_{0}+k\right)^{2}}-\frac{2}{i\left(k_{0}+k\right)^{3}}\right) \\
& -\frac{\mathcal{B}_{2}+\mathcal{B}_{3}}{2 i k} e^{-i\left(k_{0}-k\right) x} \\
& \times\left(-\frac{x^{2}}{i\left(k_{0}-k\right)}+\frac{2 x}{\left(k_{0}-k\right)^{2}}+\frac{2}{i\left(k_{0}-k\right)^{3}}\right) \\
+ & C_{20} .
\end{aligned}
$$

Substituting these solutions for $C_{1}(x)$ and $C_{2}(x)$ into formula (A3) and keeping in mind our notations and formulas 9a 9c A1b we obtain the general solution for "positive" and "negative" waves:

$$
\begin{aligned}
A_{ \pm}^{(1)}(x, \Omega)=C_{ \pm 0} e^{ \pm i \frac{\Omega}{c} x} & \\
+ & \frac{1}{2} A_{ \pm 0} h\left(\Omega-\omega_{0}\right) e^{ \pm i k_{0} x} \\
& \times\left[x^{2} \xi(\Omega) \mp i x \eta(\Omega)-\zeta(\Omega)\right] \\
& +\frac{1}{2} A_{ \pm 0}^{*} h\left(\Omega+\omega_{0}\right) e^{\mp i k_{0} x} \\
& \times\left[x^{2} \xi(-\Omega) \pm i x \eta(-\Omega)-\zeta(-\Omega)\right],
\end{aligned}
$$

Here we redefined for convenience constants $C_{10}$ and $C_{20}$ as $C_{+0}$ and $C_{-0}$ to describe separately the additions to "positive" and "negative" waves. Constants $C_{ \pm 0}$ are obtained from initial conditions (A1C):

$$
C_{ \pm 0}=\frac{1}{2} A_{ \pm 0} h\left(\Omega-\omega_{0}\right) \zeta(\Omega)+\frac{1}{2} A_{ \pm 0}^{*} h\left(\Omega+\omega_{0}\right) \zeta(-\Omega) .
$$

Substituting them into formula (A4) and performing inverse Fourier transformation we finally obtain the first order solution in time domain (8). 


\section{APPENDIX B: GENERAL RELATIVISTIC CORRECTIONS TO THE FORCE OF LIGHT PRESSURE}

In this Appendix we shall prove the statement made in Sec. VI the 4-force of light pressure $\mathcal{F}_{\mathrm{em}}^{\mu}$ can be calculated by means of classical flat space-time electrodynamics without introducing a significant error.

Whatever the general relativistic rule for calculation of the light pressure force in any case it will be proportional to: (i) the components of energy-stress tensor $T^{\alpha \beta}$ of the light field inside the cavity averaged over period of optical oscillations $2 \pi / \omega_{0}$, (ii) cross-section of the light beam $S$ and (iii) components of metric tensor (2):

$$
\mathcal{F}_{\mathrm{em}}^{\mu} \propto g_{\lambda \nu} T^{\alpha \beta} S .
$$

The energy-stress tensor of electromagnetic field in curved space time is [33]:

$$
T_{\mu \nu}=\frac{1}{4 \pi}\left(F_{\mu \alpha} F_{\nu \beta} g^{\alpha \beta}-\frac{1}{4} F_{\alpha \beta} F^{\alpha \beta} g_{\mu \nu}\right),
$$

where $F_{\alpha \beta}$ is the tensor of electromagnetic field $A^{\gamma}$ inside the cavity:

$$
\begin{aligned}
F_{\alpha \beta} & =\nabla_{\alpha} A_{\beta}-\nabla_{\beta} A_{\alpha}=g_{\beta \gamma} \nabla_{\alpha} A^{\gamma}-g_{\alpha \gamma} \nabla_{\beta} A^{\gamma} \\
& =g_{\beta \gamma}\left(\frac{\partial A^{\gamma}}{\partial x^{\alpha}}+\Gamma_{\nu \alpha}^{\gamma} A^{\nu}\right)-g_{\alpha \gamma}\left(\frac{\partial A^{\gamma}}{\partial x^{\beta}}+\Gamma_{\nu \beta}^{\gamma} A^{\nu}\right) .
\end{aligned}
$$

Here $\nabla_{\alpha}$ is the covariant derivative with respect to the connection of space-time $\Gamma_{\nu \lambda}^{\mu}$ :

$$
\Gamma_{\nu \lambda}^{\mu}=\frac{1}{2} g^{\mu \alpha}\left(\frac{\partial g_{\alpha \nu}}{\partial x^{\lambda}}+\frac{\partial g_{\alpha \lambda}}{\partial x^{\nu}}-\frac{\partial g_{\nu \lambda}}{\partial x^{\alpha}}\right)
$$

We shall now examine the orders of the values included in $F_{\alpha \beta}$. To do this we shall omit all numerical constants of the order of unity and all terms of the second and greater orders in $h$. Besides we shall consider only the case of monochromatic GW with frequency $\omega_{\mathrm{gw}}$ since generalization is trivial. Remind that all functions are evaluated at point $x=L$. Returning to Sec. [1 we conclude that

$$
g_{\mu \nu} \sim 1+\frac{L^{2}}{c^{2}} \ddot{h}, \quad \Gamma_{\nu \lambda}^{\mu} \sim \frac{L^{2}}{c^{2}} \frac{\omega_{\mathrm{gw}}}{c} \ddot{h}+\frac{L}{c^{2}} \ddot{h} .
$$

Remind that (see formula (11a) electromagnetic field $A^{\gamma}(x, t)$ inside the cavity is a sum of "positive" wave $A_{+}(x, t)$ and "negative" wave $A_{-}(x, t)$. These waves include "big" components with amplitudes $A_{ \pm 0}$ and "small" components proportional to $A_{ \pm 0} h$ (we are not interested here in the term proportional to $X$ ). It is necessary to mention that these "small" terms are included in the wavefunctions of electromagnetic field and, in general, the gravitational nature of these small corrections is of no importance to us here. Therefore, we shall not consider them as general relativistic corrections further, since we are interested only in GR corrections to the methods of flat space-time electrodynamics but not the corrections included in $A^{\gamma}(x, t)$ itself. We estimate the order of the derivative of EMW field as

$$
\left.\frac{\partial A}{\partial x}\right|_{L} \sim k_{0} A_{0}+k_{0} A_{0} H(L, t)
$$

where $H(L, t) \sim h$ is the mentioned small correction to the EMW field inside the cavity, containing GW wavefunction. Thus we can estimate the order of $F_{\alpha \beta}$ in the following way:

$$
F_{\alpha \beta} \sim k_{0} A_{0}\left(1+H(L, t)+\frac{L^{2}}{c^{2}} \ddot{h}+\frac{L^{2}}{c^{2}} \frac{\omega_{\mathrm{gw}}}{\omega_{0}} \ddot{h}+\frac{L}{\omega_{0} c} \ddot{h}\right) .
$$

Here $A_{0}$ has the order of magnitude of the electromagnetic wave's amplitude inside the cavity. The light pressure 4 -force $\mathcal{F}_{\mathrm{em}}^{\mu}$ is proportional to $F_{\alpha \beta}$, "squared" (remind that we neglect all numerical coefficients), averaged over period of optical oscillations $2 \pi / \omega_{0}$ (terms proportional to $e^{2 i \omega_{0} t}$ disappear) and multiplied by $S$ :

$$
\begin{aligned}
\mathcal{F}_{\mathrm{em}}^{\mu} \sim & W_{\mathrm{FP}} S \\
& \times\left(1+H(L, t)+\frac{L^{2}}{c^{2}} \ddot{h}+\frac{L^{2}}{c^{2}} \frac{\omega_{\mathrm{gw}}}{\omega_{0}} \ddot{h}+\frac{L}{\omega_{0} c} \ddot{h}\right),
\end{aligned}
$$

where $W_{\mathrm{FP}} \sim k_{0}^{2} A_{0}^{2}$ is proportional to the energy density of light wave inside the cavity.

Let us now return to the mirror's equation of motion:

$$
\frac{d^{2} x^{\mu}}{d s^{2}}+\Gamma_{\nu \lambda}^{\mu} \frac{d x^{\nu}}{d s} \frac{d x^{\lambda}}{d s}=\frac{1}{m} \mathcal{F}_{\mathrm{em}}^{\mu}
$$

We rewrite this equation in non-relativistic limit for $x$ component, expanding the force of light pressure into the zeroth and the first order in $h$ terms:

$$
\frac{d^{2} x}{d t^{2}}-\frac{1}{2} x \ddot{h}(t)=\frac{1}{m}\left(F^{(0)}+F_{\mathrm{FP}}^{(1)}+F_{\mathrm{GR}}^{(1)}\right),
$$

where

$$
F^{(0)} \sim W_{\mathrm{FP}} S
$$

is the constant force of light pressure;

$$
F_{\mathrm{FP}}^{(1)} \sim W_{\mathrm{FP}} S H(L, t),
$$

is the part of the force proportional to the phase shift of light due to its direct interaction with GW;

$$
F_{\mathrm{GR}}^{(1)} \sim W_{\mathrm{FP}} S\left(\frac{L^{2}}{c^{2}} \ddot{h}+\frac{L^{2}}{c^{2}} \frac{\omega_{\mathrm{gw}}}{\omega_{0}} \ddot{h}+\frac{L}{\omega_{0} c} \ddot{h}\right),
$$

is the GR correction to the methods of flat space-time electrodynamics. Our problem is to prove that it can be omitted when calculating the force of light pressure. We use the method of successive approximations to solve the equation of motion. The zeroth order force is constant, 
therefore we should redefine the length $L$ in all formulas, assuming that in real experiment constant force is compensated somehow. The first order equation is then

$$
\frac{d^{2} X}{d t^{2}}-\frac{1}{2} L \ddot{h}(t)=\frac{1}{m} F_{\mathrm{FP}}^{(1)}+\frac{1}{m} F_{\mathrm{GR}}^{(1)} .
$$

To estimate the orders of all the terms included in this equation we turn to the spectral domain:

$$
-\Omega^{2} X(\Omega)+\frac{1}{2} L \Omega^{2} h(\Omega)=\frac{1}{m} F_{\mathrm{FP}}^{(1)}(\Omega)+\frac{1}{m} F_{\mathrm{GR}}^{(1)}(\Omega) .
$$

Its solution is:

$$
\begin{aligned}
& X(\Omega) \sim L h(\Omega)+\frac{W_{\mathrm{FP}} S}{m \Omega^{2}} H(L, \Omega) \\
& \quad+\frac{W_{\mathrm{FP}} S}{m \Omega^{2}}\left(\frac{L^{2}}{c^{2}} \omega_{\mathrm{gw}}^{2}+\frac{L^{2}}{c^{2}} \frac{\omega_{\mathrm{gw}}}{\omega_{0}} \omega_{\mathrm{gw}}^{2}+\frac{L}{\omega_{0} c} \omega_{\mathrm{gw}}^{2}\right) h(\Omega) .
\end{aligned}
$$

We can replace spectral frequency $\Omega$ with $\omega_{\mathrm{gw}}$ since $h(\Omega) \sim h_{0} \delta\left(\Omega \pm \omega_{\mathrm{gw}}\right)$. Then

$$
\begin{aligned}
X(\Omega) \sim & L h(\Omega)+\frac{W_{\mathrm{FP}} S}{m \Omega^{2}} H(L, \Omega) \\
& +\frac{\mathcal{E}_{\mathrm{FP}}}{m c^{2}}\left(1+\frac{\lambda_{0}}{\lambda_{\mathrm{gw}}}+\frac{\lambda_{0}}{L}\right) \operatorname{Lh}(\Omega),
\end{aligned}
$$

where $\mathcal{E}_{\mathrm{FP}}=W_{\mathrm{FP}} S L$ is the full energy of light wave inside the cavity. We can neglect the third term in formula (B1) completely since $\mathcal{E}_{\mathrm{FP}} / m c^{2} \approx 10^{-17}\left(\mathcal{E}_{\mathrm{FP}}=20 \mathrm{~J}\right.$ and $m=40 \mathrm{~kg}$ for Advanced LIGO project), $\lambda_{0} / \lambda_{\mathrm{gw}} \sim$ $10^{-13} \div 10^{-10}$ and $\lambda_{0} / L \approx 10^{-9}$.

Now looking back, we see that the term we have just omitted corresponds to $F_{\mathrm{GR}}^{(1)}$ - the statement required to be proved.

\section{APPENDIX C: CALCULATION OF THE FORCE OF LIGHT PRESSURE IN AN EXPLICIT FORM}

In this Appendix we calculate explicitly the force of light pressure

$$
F_{x}(X, \dot{X}, t)=\frac{S}{8 \pi}\left[\frac{1}{c^{2}}\left(\frac{\partial A}{\partial t}\right)^{2}+\left(\frac{\partial A}{\partial x}\right)^{2}\right]_{x=L+X(t)}^{(1)},
$$

which is the right side of Eq. (23) (for simplicity we use notation $F_{x}$ instead of $\left.F_{x}^{(1)}\right)$.

\section{General formula}

First we calculate the force of light pressure in time domain. Summing up the derivatives of $A_{+}(x, t)$ and $A_{-}(x, t)$ (see formula (11a)), raising the sum to the second power, neglecting the terms containing $e^{2 i \omega_{0} t}$ and keeping only the first order in $h$ terms, we obtain:

$$
\begin{aligned}
&\left.\left(\frac{\partial A}{\partial x}\right)^{2}\right|_{L+X(t)}=2 i k_{0} {\left[A_{+0}\left(\frac{\partial a_{+}^{*}}{\partial x}-i k_{0} a_{+}^{*}\right)\right.} \\
&+A_{+0}\left(\frac{\partial a_{-}^{*}}{\partial x}+i k_{0} a_{-}^{*}\right) e^{2 i k_{0} L} \\
&-A_{-0}\left(\frac{\partial a_{-}^{*}}{\partial x}+i k_{0} a_{-}^{*}\right) \\
&\left.-A_{-0}\left(\frac{\partial a_{+}^{*}}{\partial x}-i k_{0} a_{+}^{*}\right) e^{-2 i k_{0} L}\right]_{L} \\
&+ \text { c.c. }
\end{aligned}
$$

Substituting formula $A_{+0}=-A_{-0} e^{-2 i \omega_{0} \tau}$ here we obtain:

$$
\begin{aligned}
\left.\left(\frac{\partial A}{\partial x}\right)^{2}\right|_{L+X(t)} & =4 i k_{0} A_{+0}\left(\frac{\partial a_{+}^{*}}{\partial x}-i k_{0} a_{+}^{*}\right)_{L} \\
& -4 i k_{0} A_{-0}\left(\frac{\partial a_{-}^{*}}{\partial x}+i k_{0} a_{-}^{*}\right)_{L}+\text { c.c. }
\end{aligned}
$$

Similar calculations lead to

$$
\left.\frac{1}{c^{2}}\left(\frac{\partial A}{\partial t}\right)^{2}\right|_{L+X(t)}=0
$$

This is not surprising since $E_{z}(L+X)=-(\dot{X} / c) H_{y}(L+$ $X$ ) (see formula (14)) and being squared leads to $X^{2}$. Therefore, $E_{z}^{2}$ equals to zero in our linear approximation.

Substituting formula (11b) into (C1) we obtain the final formula for $F_{x}$ :

$$
F_{x}(X, \dot{X}, t)=\int_{-\infty}^{+\infty} F_{x}(\Omega) e^{-i \Omega t} \frac{d \Omega}{2 \pi},
$$

$$
\begin{aligned}
F_{x}(\Omega)=\frac{S k_{0}}{2 \pi}\left[A_{+0}^{*} a_{+}\left(\omega_{0}+\Omega\right) \frac{\omega_{0}+\Omega}{c} e^{i \Omega \tau}\right. \\
+A_{+0} a_{+}^{*}\left(\omega_{0}-\Omega\right) \frac{\omega_{0}-\Omega}{c} e^{i \Omega \tau} \\
+A_{-0}^{*} a_{-}\left(\omega_{0}+\Omega\right) \frac{\omega_{0}+\Omega}{c} e^{-i \Omega \tau} \\
\left.+A_{-0} a_{-}^{*}\left(\omega_{0}-\Omega\right) \frac{\omega_{0}-\Omega}{c} e^{-i \Omega \tau}\right] .
\end{aligned}
$$

Now we substitute explicit formulas (17a) and (17b) here and split $F_{x}(\Omega)$ into several parts according to their physical sense:

$$
F_{x}(\Omega)=F_{\text {fluct }}(\Omega)+F_{\mathrm{pm}}(\Omega)+F_{\mathrm{gw}+\mathrm{emw}}(\Omega) .
$$

Note that we have not taken into account the terms proportional to $A_{ \pm 0} g_{ \pm}(x, t)$ while calculating the force of light pressure. It is straightforward to verify, using similar calculations, that the corresponding correction can be estimated as $\delta F_{x}(\Omega) \approx S W_{\mathrm{FP}}(\Omega \tau)^{2} h(\Omega)$, resulting in inaccuracy $\delta X(\Omega) \approx\left(\mathcal{E}_{\mathrm{FP}} / m c^{2}\right) L h(\Omega) \approx 10^{-17} \operatorname{Lh}(\Omega)$. 


\section{Fluctuating force}

Here we obtain formula for the fluctuating part $F_{\text {fluct }}(\Omega)$ of the radiation pressure arising due to fluctuations $a_{\text {in }}(x, t)$ of the input light wave:

$$
\begin{aligned}
& F_{\text {fluct }}(\Omega) \\
& =\frac{S k_{0}}{2 \pi}\left[a_{\mathrm{in}}\left(\omega_{0}+\Omega\right) \frac{A_{+0}^{*} T e^{i \Omega \tau}}{1-R e^{2 i\left(\omega_{0}+\Omega\right) \tau}} \frac{\omega_{0}+\Omega}{c}\right. \\
& +a_{\mathrm{in}}^{*}\left(\omega_{0}-\Omega\right) \frac{A_{+0} T e^{i \Omega \tau}}{1-R e^{-2 i\left(\omega_{0}-\Omega\right) \tau}} \frac{\omega_{0}-\Omega}{c} \\
& -a_{\mathrm{in}}\left(\omega_{0}+\Omega\right) \frac{A_{-0}^{*} T e^{2 i\left(\omega_{0}+\Omega\right) \tau} e^{-i \Omega \tau}}{1-R e^{2 i\left(\omega_{0}+\Omega\right) \tau}} \frac{\omega_{0}+\Omega}{c} \\
& \left.-a_{\mathrm{in}}^{*}\left(\omega_{0}-\Omega\right) \frac{A_{-0} T e^{-2 i\left(\omega_{0}-\Omega\right) \tau} e^{-i \Omega \tau}}{1-R e^{-2 i\left(\omega_{0}-\Omega\right) \tau}} \frac{\omega_{0}-\Omega}{c}\right] .
\end{aligned}
$$

Using formulas (16a) and (16b) we obtain:

$$
\begin{aligned}
& F_{\text {fluct }}(\Omega) \\
& =\frac{S k_{0}}{\pi c}\left[\frac{T^{2} A_{\mathrm{in} 0}^{*}}{1-R e^{-2 i \omega_{0} \tau}} \frac{\left(\omega_{0}+\Omega\right) e^{i \Omega \tau}}{1-R e^{2 i\left(\omega_{0}+\Omega\right) \tau}} a_{\mathrm{in}}\left(\omega_{0}+\Omega\right)\right. \\
& \left.+\frac{T^{2} A_{\mathrm{in} 0}}{1-R e^{2 i \omega_{0} \tau}} \frac{\left(\omega_{0}-\Omega\right) e^{i \Omega \tau}}{1-R e^{-2 i\left(\omega_{0}-\Omega\right) \tau}} a_{\mathrm{in}}^{*}\left(\omega_{0}-\Omega\right)\right] .
\end{aligned}
$$

\section{Ponderomotive force}

Here we obtain the formula for the coefficient $\mathcal{K}(\Omega)$ and corresponding ponderomotive force $F_{\mathrm{pm}}(\Omega)=$ $-\mathcal{K}(\Omega) X(\Omega)$ (see formulas (24) and (25)). Ponderomotive force is the sum of all the terms in formula (C2) that include phase shift (18) arising due to mirror's displacement $X$ (see formulas (17a) and $(17 \mathrm{~b})$ ):

$$
\begin{aligned}
F_{\mathrm{pm}}(\Omega) & \\
=\frac{S W_{\mathrm{FP}}}{2 k_{0}} & {\left[\frac{R e^{2 i \omega_{0} \tau} e^{i \Omega \tau}}{1-R e^{2 i\left(\omega_{0}+\Omega\right) \tau}}+\frac{e^{-i \Omega \tau}}{1-R e^{2 i\left(\omega_{0}+\Omega\right) \tau}}\right] } \\
& \times \frac{\omega_{0}+\Omega}{c} i \delta \Psi_{\operatorname{mir}}(\Omega) \\
-\frac{S W_{\mathrm{FP}}}{2 k_{0}} & {\left[\frac{R e^{-2 i \omega_{0} \tau} e^{i \Omega \tau}}{1-R e^{-2 i\left(\omega_{0}-\Omega\right) \tau}}+\frac{e^{-i \Omega \tau}}{1-R e^{-2 i\left(\omega_{0}-\Omega\right) \tau}}\right] } \\
& \times \frac{\omega_{0}-\Omega}{c} i \delta \Psi_{\mathrm{mir}}^{*}(-\Omega)
\end{aligned}
$$

Substituting formula (18) here we obtain the following formula for $\mathcal{K}(\Omega)$ :

$$
\begin{aligned}
\mathcal{K}(\Omega)=-i S W_{\mathrm{FP}}[ & \frac{1+R e^{2 i\left(\omega_{0}+\Omega\right) \tau}}{1-R e^{2 i\left(\omega_{0}+\Omega\right) \tau} \frac{\omega_{0}+\Omega}{c}} \\
& \left.-\frac{1+R e^{-2 i\left(\omega_{0}-\Omega\right) \tau}}{1-R e^{-2 i\left(\omega_{0}-\Omega\right) \tau}} \frac{\omega_{0}-\Omega}{c}\right]
\end{aligned}
$$

It will be convenient for the future prospects to divide $\mathcal{K}(\Omega)$ into two parts: $\mathcal{K}(\Omega)=K(\Omega)-2 i \Omega \Gamma(\Omega)$ using the $\left(\omega_{0} \pm \Omega\right) / c$ multipliers. The first part $K(\Omega)$ is proportional to $\omega_{0} / c$ and is called the coefficient of optical rigidity:

$$
K(\Omega)=\frac{4 k_{0} S W_{\mathrm{FP}} R e^{2 i \Omega \tau} \sin 2 \omega_{0} \tau}{1-2 R e^{2 i \Omega \tau} \cos 2 \omega_{0} \tau+R^{2} e^{4 i \Omega \tau}},
$$

The second part $-2 i \Omega \Gamma(\Omega)$ is proportional to $\Omega / c$ where $\Gamma(\Omega)$ is called the coefficient of radiative friction:

$$
\Gamma(\Omega)=\frac{S W_{\mathrm{FP}}}{c} \frac{1-R^{2} e^{4 i \Omega \tau}}{1-2 R e^{2 i \Omega \tau} \cos 2 \omega_{0} \tau+R^{2} e^{4 i \Omega \tau}} .
$$

Therefore, we secure full analogy with linear harmonic oscillator's mechanical impedance. Obviously, $|2 \Omega \Gamma| \sim$ $\left(\Omega / \omega_{0}\right)|K|$.

\section{GW+EMW correction}

Here we obtain formula (26) for the 'GW+EMW' correction to the force of light pressure. It arises due to direct interaction of gravitational wave with the light wave inside the cavity and becomes significant when length of the cavity becomes comparable with gravitational wavelength. Therefore, this part of (C2) is composed of the terms that include phase shift (19) arising due to direct coupling 'GW+EMW':

$$
\begin{aligned}
& F_{\text {gw }+ \text { emw }}(\Omega) \\
& =\frac{S W_{\mathrm{FP}}}{2 k_{0}}\left[\frac{R e^{2 i \omega_{0} \tau} e^{i \Omega \tau}}{1-R e^{2 i\left(\omega_{0}+\Omega\right) \tau}}+\frac{e^{-i \Omega \tau}}{1-R e^{2 i\left(\omega_{0}+\Omega\right) \tau}}\right] \\
& \times \frac{\omega_{0}+\Omega}{c} i \delta \Psi_{\mathrm{gw}+\mathrm{emw}}(\Omega) \\
& -\frac{S W_{\mathrm{FP}}}{2 k_{0}}\left[\frac{R e^{-2 i \omega_{0} \tau} e^{i \Omega \tau}}{1-R e^{-2 i\left(\omega_{0}-\Omega\right) \tau}}+\frac{e^{-i \Omega \tau}}{1-R e^{-2 i\left(\omega_{0}-\Omega\right) \tau}}\right] \\
& \times \frac{\omega_{0}-\Omega}{c} i \delta \Psi_{\mathrm{gw}+\mathrm{emw}}^{*}(-\Omega) \text {. }
\end{aligned}
$$

Substituting formula (20) here we obtain:

$$
\begin{aligned}
F_{\mathrm{gw}+\mathrm{emw}}(\Omega)= & -\frac{1}{2} \operatorname{Lh}(\Omega)\left(1-\frac{\sin \Omega \tau}{\Omega \tau}\right) i S W_{\mathrm{FP}} \\
\times & {\left[\frac{1+R e^{2 i\left(\omega_{0}+\Omega\right) \tau}}{1-R e^{2 i\left(\omega_{0}+\Omega\right) \tau} \frac{\omega_{0}+\Omega}{c}}\right.} \\
& \left.-\frac{1+R e^{-2 i\left(\omega_{0}-\Omega\right) \tau}}{1-R e^{-2 i\left(\omega_{0}-\Omega\right) \tau}} \frac{\omega_{0}-\Omega}{c}\right]
\end{aligned}
$$

Taking into account formula (C3) we finally obtain the following formula for the 'GW+EMW' correction:

$$
F_{\mathrm{gw}+\mathrm{emw}}(\Omega)=\frac{1}{2} \mathcal{K}(\Omega)\left(1-\frac{\sin \Omega \tau}{\Omega \tau}\right) \operatorname{Lh}(\Omega),
$$

which coincides with (26). 
[1] R. Weiss, Quarterly Progress Report, Research Lab. of Electronics, M.I.T. 105, 54 (1972).

[2] A. Abramovici et al., Science 256, 325 (1992).

[3] L. Ju, D.G. Blair and C. Zhao, Rep. Prog. Phys. 63, 1317 (2000).

[4] E.E. Flanagan and S.A. Hughes, New J. Phys. 7, 204 (2005), arXiv:gr-qc/0501041.

[5] R. Blandford and K.S. Thorne, Ph 136: Applications of Classical Physics (California Institute of Technology, Pasadena, 2003), chap. 26, URL http://www.pma.caltech.edu/Courses/ph136/yr2002/ch

[6] C. Misner, K. Thorne and J. Wheeler, Gravitation, vol. 3 (San Francisco, W.H. Freeman and Company, 1973); C. Misner, K. Thorne and J. Wheeler, Gravitation, vol. 3 (Moskva, Mir, 1977).

[7] V.B. Braginsky and A.B. Manukin, Sov. Phys. JETP 25, 653 (1967); V.B. Braginsky and A.B. Manukin, ZhETF 52, 986 (1967).

[8] V.B. Braginsky, A.B. Manukin and M.Yu. Tikhonov, Sov. Phys. JETP 31, 829 (1970); V.B. Braginsky, A.B. Manukin and M.Yu. Tikhonov, ZhETF 58, 1550 (1970).

[9] V.B. Braginsky, M.L. Gorodetsky and F.Ya. Khalili, Phys. Lett. A 232, 340 (1997).

[10] V.B. Braginsky and F.Ya. Khalili, Phys. Lett. A 257, 241 (1999).

[11] M. Rakhmanov, Ph.D. thesis, California Institute of Technology (2000), URL http://www. ligo.caltech.edu/docs/P/P000002-00.pdf

[12] F.Ya. Khalili, Phys. Lett. A 288, 251 (2001), arXiv:grqc/0107084.

[13] A. Buonanno and Y. Chen, Phys. Rev. D 64, 042006 (2001), arXiv:gr-qc/0102012.

[14] A. Buonanno and Y. Chen, Phys. Rev. D 65, 042001 (2002), arXiv:gr-qc/0107021.

[15] A. Buonanno and Y. Chen, Phys. Rev. D 67, 062002 (2003), arXiv:gr-qc/0208048.

[16] V.I. Lazebny and S.P. Vyatchanin, Phys. Lett. A 344, 7 (2005).

[17] F.Ya. Khalili, V.I. Lazebny and S.P. Vyatchanin, Phys. Rev. D 73, 062002 (2006), arXiv:gr-qc/0511008.

[18] A.B. Matsko, E.A. Zubova and S.P. Vyatchnin, Opt. Commun. 131, 107 (1996).

[19] C. Cutler, Phys. Rev. D 57, 7089 (1998), arXiv:gr- qc/9703068

[20] T.A. Prince et al., Phys. Rev. D 66, 122002 (2002), arXiv:gr-qc/0209039.

[21] M. Rakhmanov, Phys. Rev. D 71, 084003 (2005), arXiv:gr-qc/0406009.

[22] M. Maggiore, Phys. Rep. 331, 283 (2000), arXiv:grqc/9909001.

[23] B. Allen and J.D. Romano, Phys. Rev. D 59, 102001 (1999), arXiv:gr-qc/9710117.

[24] M. Maggiore, Lecture given at the: Gravitational Waves: 26/0226Cliapdef to Theoretical Astrophysics. Trieste, 5-9 June 2000, arXiv:gr-qc/0008027.

[25] R. Brustein et al., Phys. Lett. B 361, 45 (1995), arXiv:hep-th/9507017.

[26] B. Allen and R. Brustein, Phys. Rev. D 55, 3260 (1997), arXiv:gr-qc/9609013.

[27] A. Buonanno, M. Maggiore and C. Ungarelli, Phys. Rev. D 55, 3330 (1997), arXiv:gr-qc/9605072.

[28] J. Markowicz, R. Savage and P. Schwinberg, LIGO technical document T030186-00 (2003), URL http://www.ligo.caltech.edu/docs/T/T030186-00.pdf

[29] D. Sigg and R. Savage, LIGO technical document T030296-00 (2003), URL http://www.ligo.caltech.edu/docs/T/T030296-00.pdf

[30] W.E. Buttler, Ph.D. thesis, University of Rochester (2004), URL http://www.ligo.caltech.edu/docs/P/P040026-00.pdf

[31] M. Rakhmanov et al., Class. Quantum Grav. 21, S487 (2004).

[32] R. Schilling, Class. Quantum Grav. 14, 1513 (1997).

[33] L. Landau and E. Lifshitz, The Classical Theory of Fields (New York, Pergamon Press, 1971); L.D. Landau and E.M. Lifshitz, The Classical Theory of Fields, vol. 2 of Theoretical Physics, (Moskva, Fizmatlit, 2001).

[34] C.K. Law, Phys. Rev. A 51, 2537 (1995).

[35] D. Baskaran and L.P. Grishchuk, Class. Quantum Grav. 21, 4041 (2004).

[36] C. Corda, arXiv:gr-qc/0610156.

[37] V.B. Braginsky, S.E. Strigin and S.P. Vyatchanin, Phys. Lett. A 287, 331 (2001), arXiv:gr-qc/0107079.

[38] V.B. Braginsky, S.E. Strigin and S.P. Vyatchanin, Phys. Lett. A 305, 111 (2002), arXiv:gr-qc/0209064. 